\title{
The Structure of Scientific Theories, Explanation, and Unification. A Causal-Structural Account
}

\author{
Bert Leuridan
}

Forthcoming in The British Journal for the Philosophy of Science.

\begin{abstract}
What are scientific theories and how should they be represented? In this paper I propose a causal-structural account, according to which scientific theories are to be represented as sets of interrelated causal and credal nets. In contrast with other accounts of scientific theories (such as Sneedian structuralism, Kitcher's unificationist view, and Darden's theory of theoretical components), this leaves room for causality to play a substantial role. As a result, an interesting account of explanation is provided which sheds light on explanatory unification within a causalist framework. The theory of classical genetics is used as a case study.
\end{abstract}

1 Introduction

2 The Theory of Classical Genetics

3 Three Philosophical Accounts of the Theory of Classical Genetics

3.1 The structuralist account

3.2 Kitcher's unificationism

3.3 Darden and theory change in science 
4 A Common Lacuna: Where is Causality?

$5 \quad$ Woodward's Interventionist Account of Causation

$6 \quad$ Causal Bayes Nets and Their Interrelations

6.1 Causal Bayes nets

6.2 Relations among causal nets

6.3 Credal nets and their interrelations

$7 \quad$ The Theory of the Gene and its Causal Graph

8 A First Exemplar: Stem Length in Pea Plants

8.1 Three crosses on stem length in pea plants

8.2 The causal graph for stem length in pea plants

8.3 Morgan's explanatory principles and the credal net for stem length in pea plants

$9 \quad$ Explaining Mendel's Crosses: a Causal-Structural Account

10 Monohybrid Crosses with Complete Dominance

11 Exemplars, Abstract Explanatory Patterns, Generic Credal Nets and Mechanism Schemas

12 Incomplete Dominance

$13 \quad$ Anomalies

$14 \quad$ Multihybrid Crosses with Independent Assortment

$15 \quad$ Multihybrid Crosses with Linkage and Crossing-over

16 Double Crossing-over and the Linear Order of the Gene

17 Causal-Structural Explanation

18 Explanatory Unification

19 Concluding Remarks 


\section{Introduction}

What are scientific theories and how should they be represented? In this paper, I propose a causal-structural account according to which scientific theories are to be represented as sets of interrelated causal and credal nets. The theory of classical genetics will be used as a case study and several philosophical topics (relating to exemplars, anomalies, explanation and unification) will be explored.

What scientific theories are and how they should be represented, have been very pressing questions in 20th century philosophy of science. In search for an answer, many have used the theory of classical genetics as a case study. Woodger ([1929], [1952], [1959]) has provided an elaborate reconstruction of classical genetics within the neopositivist axiomatic framework. Balzer and Lorenzano ([2000]) and others have discussed classical genetics extensively within the Sneedian structuralist framework (based on Balzer et al. [1987]). ${ }^{1}$ Others have used the case of classical genetics to address related questions about theory change in science (Darden [1991]), or about explanation and unification (Kitcher [1989]).

Unfortunately, causality plays no substantial role in any of the above accounts. During the past decades, however, more and more philosophers have stressed the centrality of causality in, among other things, explanation. If they are correct, which I think they are, and if scientific theories can be used for explanation, which I think they can, then we need an account of scientific theories that allows us to represent their causal structure (see section 4 for a more elaborate discussion of causality and explanation). It will emerge that my causal-structural approach, which builds on Woodward's interventionist account of causation and on the theory of causal Bayes nets, is very suitable to that task. It naturally 
represents both the qualitative and the quantitative aspects of the causal structure of classical genetics in an integrated way. It also results in an interesting account of explanation which allows us to get a grip on explanatory unification within a causalist framework. Moreover, it offers a nice explication of lawfulness in classical genetics and in the special sciences in general (see section 17). A final advantage, which I will not stress here, is that it can easily be implemented in computers and AI; see the tools developed by Spirtes et al. ([2000]), such as TETRAD. Some of the leading figures discussed in this paper have endeavoured a similar advantage in the past, using different tools such as PROLOG (Balzer and Lorenzano [2000], p. 243) and SUTTON (Darden and Rada [1988]).

This paper consists of three parts. In the first part (sections 2-6) I will set the stage. In section 2 I introduce the reader to the main concepts of classical genetics. In section 3 , I briefly present three existing philosophical accounts of classical genetics, by the structuralists, by Kitcher and by Darden. Their ideas will play an important role in this paper and I will try to accommodate several of them. Yet I will also point to a very important lacuna they in common: they have no substantial role for causality to play (section 4). Finally, I will sketch Woodward's interventionist account of causation (section 5) and the basic formal concepts to be used in this paper: causal nets, credal nets, and several kinds of isomorphism relations (section 6).

In the second part (sections 7-16), I will present the causal-structural account of scientific theories by applying it to the theory of classical genetics. More specifically, I present classical genetics' qualitative causal structure (section 7), its application to monohybrid crosses with complete dominance (sections 8 and 10) and incomplete dominance (section 12), and 
its application to multihybrid crosses with independent assortment (section 14) and with linkage and crossing over (sections 15-16). Meanwhile, I also present a causal-structural account of explanation (section 9) and glance at Darden's discussion of exemplars (section 11) and anomalies (section 13).

In the third part (sections 17-18), I will expand on the causal-structural account of scientific explanation (section 17), and highlight one of its important advantages, viz. that it allows us to get a grip on explanatory unification within a causalist framework (section 18).

Finally, I conclude in section 19 by discussing the possible generalizability of the causal-structural account of scientific theories.

\section{The Theory of Classical Genetics}

The theory of classical genetics has been developed roughly between 1900 and the end of the 1920's. In the middle of the 19th century, Gregor Mendel studied phenomena of inheritance and hybridization mainly by experimenting with pea plants. He presented his seminal ideas in 1865 . In 1866 these were published in his Versuche über Pflanzen-Hybriden (Mendel [1865], [1933]). In 1900, his work again came to the fore, due to the works of De Vries ([1900]), Correns ([1900]) and, shortly afterwards, Bateson ([1900], [1902]). Almost thirty years later, Thomas Hunt Morgan published the second edition of The Theory of the Gene (1928). This can be considered an end-point in the development of classical genetics (Darden [1991], pp. 3 and 38). In this paper, I will use Morgan's exposition as my main source for exploring the structure of classical genetics. But first I will introduce the main concepts of classical genetics based on a contemporary textbook (Klug et al. [2006]).

The main aim of classical genetics is to explain the distribution of 
observable traits in the offspring of two (groups of) crossed organisms (Klug et al. [2006], chapters 3-5). For example, why is it that after crossing true-breeding tall pea plants with short pea plants all the resulting offspring is tall? ${ }^{2}$ And why is it that after crossing this offspring with short pea plants, half of the resulting plants are tall whereas the other half are short?

Traits (such as tall and short) are alternative forms of the same character (in casu stem length in pea plants). (Klug et al. [2006], p. 40) They are assumed to be coded for (or caused) by the organism's genes. Alleles are alternative forms of genes. (Klug et al. [2006], p. 42) For example, there exists an allele for tall stems and an allele for short stems. (Other genes may have more than two possible alleles; Klug et al. [2006], section 4.5.) An organism's observable features are called its phenotype; the set of its alleles for a given character (or set of characters) is called its genotype. (Klug et al. [2006], p. 42) In their experiments, classical geneticists focussed on the transmission of one character (monohybrid crosses), or more characters (dihybrid, trihybrid, ... crosses). (Klug et al. [2006], pp. 40-4) The number of copies an organism has of each gene depends on its number of chromosomes. In this paper, I will focus on diploid organisms. These have two copies of each chromosome, and hence two copies of each gene. (Klug et al. [2006], p. 20) If both copies are identical (the same allele), the individual is homozygous, otherwise it is heterozygous. (Klug et al. [2006], p. 42) The specific location of a specific gene on its chromosome is called its locus (plural: loci) (Klug et al. [2006], p. 51).

Alleles are transmitted to the next generation via the gametes or germ-cells. Barring cases of crossing-over, each germ-cell receives one 
member of each pair of chromosomes, and hence one copy of each gene (Klug et al. [2006], pp. 27-30). By invoking certain hypotheses about this process, the distribution of genotypes (and hence of phenotypes) in the offspring can be explained. With respect to each pair of alleles, the law of segregation states that during gamete formation, the paired alleles separate or segregate randomly, so that each germ-cell receives one or the other with equal likelihood. (Klug et al. [2006], p. 42) When multiple pairs of alleles are investigated (as is the case in multihybrid crosses) extra assumptions are needed. Mendel assumed that the transmission of one pair of alleles was independent of the transmission of other pairs. This was later called the law of independent assortment (Klug et al. [2006], p. 46). This law holds only for genes that lie on different chromosomes. Genes lying on the same chromosome are said to be linked (Klug et al. [2006], p. 101). Normally, gametes do not receive exact copies of the original chromosomes. Homologous chromosomes (chromosomes of the same pair) typically exchange part of their material (crossing-over). As a result, linkage is normally incomplete (Klug et al. [2006], p. 101).

Other hypotheses pertain to the relation between genotype and phenotype. The best-known principle is complete dominance. For example, a pea plant that is heterozygous for stem length is tall (the tall-allele is dominant, the short-allele is recessive). Not all characters show complete dominance, however (see section 12).

\section{Three Philosophical Accounts of the Theory of Classical Genetics}

In this section, I will briefly present three existing philosophical accounts of the theory of classical genetics: by the structuralists, by Kitcher and by 
Darden. Even though they have an important problem in common (they all treat issues of causality in stepmotherly fashion; see section 4), these three accounts definitely deserve our attention. I will focus on the structuralist notions of 'theory-element', 'T-theoreticity' and explanation as 'embedding', on Kitcher's 'general argument patterns' and on Darden's notions of 'theoretical component' and 'abstract explanatory pattern'. In the rest of this paper, these notions will be translated into the language of causal Bayes nets. This will prove to be fruitful in the construction of an adequate account of scientific theories, of explanation and of unification. ${ }^{3}$

\subsection{The structuralist account}

Balzer Lorenzano ([2000]) have analysed the theory of classical genetics within the Sneedian structuralist framework, in which mature scientific theories are represented in terms of their logical models, i.e. set-theoretic structures that satisfy particular statements or propositions (Balzer et al. [1987], pp. 2-3). In the structuralist approach, scientific theories are rarely treated as monolithic entities. They are usually represented as theory-nets: partially ordered sets of theory-elements, which are the basic building blocks of scientific theories (Balzer et al. [1987], p. 172). A theory-element $\mathbf{T}=\langle\mathbf{K}, \mathbf{I}\rangle$ consists of a core $\mathbf{K}$ and a limited domain of intended applications I (Balzer et al. [1987], p. 39).

The theory-core $\mathbf{K}(\mathbf{T})=\left\langle\mathbf{M}_{\mathbf{p}}(\mathbf{T}), \mathbf{M}(\mathbf{T}), \mathbf{M}_{\mathbf{p p}}(\mathbf{T}), \mathbf{G C}(\mathbf{T}), \mathbf{G L}(\mathbf{T})\right\rangle$ consists of five elements (Balzer et al. [1987], p. 79). $\mathbf{M}_{\mathbf{p}}(\mathbf{T})$, the set of $\mathbf{T}$ 's potential models, consists of those set-theoretic structures that can be subsumed under T's conceptual framework; i.e. the structures for which it makes sense to ask whether they satisfy T's laws (Balzer et al. [1987], pp. 15-7). $\mathbf{M}(\mathbf{T})$ is the set of $\mathbf{T}$ 's models, i.e. those potential models that 
satisfy T's laws (hence $\mathbf{M}(\mathbf{T}) \subset \mathbf{M}_{\mathbf{p}}(\mathbf{T})$ ) (Balzer et al. [1987], pp. 3 and 15-7). $\mathbf{M}_{\mathbf{p p}}(\mathbf{T})$ is the set of $\mathbf{T}$ 's partial potential models, i.e. those fragments of the potential models which contain only T's non-theoretical conceptual apparatus (Balzer et al. [1987], pp. 56-7). Roughly speaking, a concept is $\mathbf{T}$-non-theoretical if there are ways of determining or measuring that concept which do not invoke T's laws, and T-theoretical otherwise (Balzer et al. [1987], pp. 50ff). For example, in classical genetics, 'phenotype' is a non-theoretical concept; 'genotype' is theoretical (see section 7). ${ }^{4}$ Partial potential models can be 'extended' to potential models by adding suitable $\mathbf{T}$-theoretical relations (Balzer et al. [1987], pp. 56-7). T's constraints, $\mathbf{G C}(\mathbf{T})$, characterize the connections among the different local applications of T (Balzer et al. [1987], pp. 40-1 and 78). For example, one constraint may state that the same genotype used in two different models will produce the same phenotype in these respective models (Balzer and Lorenzano [2000], p. 254). Finally, T's intertheoretical links, $\mathbf{G L}(\mathbf{T})$, represent the transfer of data from another theory $\mathbf{T}^{\prime}$ to $\mathbf{T}$ (Balzer et al. [1987], pp. 58 and 79). Such transfer is needed for measuring T-non-theoretical concepts.

The set of intended applications $\mathbf{I}$ describes the phenomena that $\mathbf{T}$ should account for (Balzer et al. [1987], pp. 86-9). The intended applications have the structure of partial potential models. But $\mathbf{M}_{\mathbf{p p}}$ contains all of T's possible applications (even 'purely mathematical' structures), not all of which are intended; therefore, $\mathbf{I} \subseteq \mathbf{M}_{\mathbf{p p}}$. The distinction between merely possible applications and intended possible applications cannot be made in a purely formal way (Balzer et al. [1987], pp. 88-9). Often, the set of intended applications is specified by citing a few 'paradigms' or 'exemplars' (such as 'the pendulum' and 'the projectile' 
in classical particle mechanics). Systems that are sufficiently similar to these exemplars are then said to belong to $\mathbf{I}$.

To explain some given data or phenomena by means of $\mathbf{T}$, one proceeds as follows (Balzer et al. [1987], p. 23). By assumption, the data have the structure of one of T's partial potential models (some $\left.M_{p p} \in \mathbf{M}_{\mathbf{p p}}\right)$. By adding $\mathbf{T}$ 's theoretical concepts, one creates a potential model (some $M_{p} \in \mathbf{M}_{\mathbf{p}}$ ); this 'embedding' of the data in a potential model is the conceptual aspect of the application of $\mathbf{T}$. Then one asserts that the data satisfy the laws of $\mathbf{T}$, i.e. that $M_{p}$ is an actual model of $\mathbf{T}\left(M_{p} \in \mathbf{M}\right)$. This assertion has empirical consequences which, if they turn out to be true, show that we have successfully applied $\mathbf{T}$ to $\mathbf{I}$.

To simplify matters, Balzer and Lorenzano do not distinguish between actual, potential and partial potential models. In their reconstruction, all the models of classical genetics consist of eight components: a set $J$ of genetic individuals (these are individual organisms or populations thereof), a set $P$ of phenotypes, a set $G$ of genotypes, and five functions relating these sets to one another (e.g., APPEARANCE is a function mapping individuals to their phenotypes) ([2000], pp. 246-9). Different theory-elements are obtained by specifying the number of genes that are investigated, or by specifying the precise mathematical form of the models' functions. They distinguish, among other things, between theory-elements for monohybrid crosses with complete dominance, for monohybrid crosses with incomplete dominance, for dihybrid crosses with independent assortment, etc.; together, these theory-elements form the theory-net of classical genetics ([2000], pp. 260-2). Data are explained by embedding them in one of classical genetics' models ([2000], p. 3).

Like Balzer and Lorenzano, I will discuss the different 
'theory-elements' of classical genetics separately and I will explore their formal interrelations (albeit using a different formal apparatus). I will also adopt the notion of 'T-theoreticity' and of explanation as 'embedding'. These ideas, together with some of Kitcher's and Darden's, will help to get an adequate grip on explanatory unification.

\subsection{Kitcher's unificationism}

Kitcher's main research question is not how to represent scientific theories, but how to build a unificationist theory of explanation.

According to Kitcher ([1989], p. 447), scientific theories are constituted by patterns of derivation or general argument patterns, which are defined as follows: ${ }^{5}$

A schematic sentence is an expression obtained by replacing some, but not necessarily all, the nonlogical expressions occurring in a sentence with dummy letters. [...] A set of filling instructions for a schematic sentence is a set of directions for replacing the dummy letters of the schematic sentence, such that, for each dummy letter, there is a direction that tells us how it should be replaced. [...] A schematic argument is a sequence of schematic sentences. A classification for a schematic argument is a set of statements describing the inferential characteristics of the schematic argument: it tells us which terms of the sequence are to be regarded as premises, which are inferred from which, what rules of inference are used, and so forth. Finally, a general argument pattern is a triple consisting of a schematic argument, a set of sets of filling instructions and a classification for the schematic argument. 
(Kitcher [1989], p. 432, original emphasis)

Kitcher ([1989], pp. 438-42) characterizes classical genetics as a set of patterns that serve to answer specific types of questions:

What is the expected distribution of phenotypes in a particular generation? Why should we expect to get that distribution?

What is the probability that a particular phenotype will result from a particular mating?, and so forth. (Kitcher [1989], p. 438)

The general idea common to all patterns is that these questions may be answered

[...] by making hypotheses about the relevant genes, their phenotypic effects, and their distribution among the individuals in the pedigree. (Kitcher [1989], pp. 438-9)

The simplest pattern, for example, is called Mendel and has four premises (Kitcher [1989], p. 439). The first premise says that there are two alleles $A$ and $a$, where $A$ is dominant and $a$ recessive. It is a schematic sentence with $A$ and $a$ as dummy letters. The filling instructions state that these are to be replaced with names of alleles. The second premise says that $A A$ and $A a$ individuals have trait $P$, whereas $a a$ individuals have trait $P^{\prime}$. The dummy letters $P$ and $P^{\prime}$ are to be replaced with names of phenotypic traits. The third premise is a list specifying the genotypes of all the individuals involved. And the fourth premise says that 'For any individual $x$ and any alleles $y z$ if $x$ has $y z$ then the probability that $x$ will transmit $y$ to any one of its offspring is $1 / 2$ ' (Kitcher [1989], p. 439). This is the law of segregation. From these premises, the expected distribution of progeny phenotypes can be derived by filling in the dummy letters. Mendel is 
limited to cases with only one gene and two possible alleles, and with complete dominance. Other, more complicated patterns (called Refined Mendel and Morgan) are more encompassing (Kitcher [1989], pp. 440-1).

\subsection{Darden and theory change in science}

Darden's main research question is how scientific theories change over time, how new scientific ideas are developed, and what strategies there are for theory change in science (Darden [1991]). To that end, she analyses the theory of classical genetics in terms of theoretical components, 'parts of the theory that change over time' (Darden [1991], p. 18). For example, between 1900 and 1903 Mendelian genetics incorporated the following component:

Unit-characters: (1) An organism is to be viewed as composed of separable unit-characters. (Adapted from Darden [1991], p. 168)

This component gradually evolved. Where, according to Darden, no distinction was made in 1900-1903 between observable traits and underlying elements or factors, that distinction was evident as of 1910:

Factors and characters. (1') Characters are produced by factors. (2a') One factor may produce one character or (2b') multiple factors may interact in the production of one character. (Adapted from Darden [1991], p. 168)

By 1926, this evolved into the following:

Genes and characters: (1") Genes cause characters. (2a") One gene may cause one character or (2b") multiple factors (genes at different loci in linkage groups) may interact in causing one 
character or (2c") one gene may affect many characters.

(Adapted from Darden [1991], p. 169)

Theoretical components are not the same as structuralist theory-elements. First, a single theory-element may (and usually does) satisfy more than one law or explanatory principle. Theoretical components are such explanatory principles. Hence theory-elements consist of more than one theoretical component. (In section 17, I will expand on my views on laws and explanatory principles.) Second, whereas theoretical components are inherently dynamic, theory-elements are more or less stable end-products of such theory evolution (but see Balzer et al. [1987], chapter V, for an account of the diachronic structure of theories). Darden's analogue of a theory-element is an abstract explanatory pattern - a notion derived from Kitcher. Each pattern invokes a number of theoretical components and is introduced by means of one or more exemplars or paradigmatic crosses (see section 11).

\section{A Common Lacuna: Where is Causality?}

These three accounts of the theory of classical genetics have one lacuna in common: to a greater or lesser extent, they treat issues of causality in stepmotherly fashion.

First, Balzer et al. ([1987]) do not discuss the difference between causal and non-causal relations or laws. It could well be that (some of) a theory's laws are causal, but the structuralist framework lacks the expressive power to say so. As a result, the structuralist account of explanation (see section 3.1) is a-causal. Moreover, it is also intended as an account of prediction; no distinction is made between prediction and explanation. And even when they apply their framework to overtly causal theories such as 
classical genetics, the structuralists are reluctant to make room for causality. Balzer and Lorenzano, for example, talk about phenotypes being 'caused' (with quotation marks!) by genotypes ([2000], pp. 247-8). ${ }^{6}$

A notable exception in the structuralist literature is Forge ([2002]), who goes some way to giving a structuralist account of causal explanation. He conjectures that in structuralist reconstructions of scientific theories, causes can be represented by (unconstrained) theoretical concepts or functions ([2002], p. 113). While this conjecture is interesting, two remarks should be made. First, Forge focuses on scientific theories in the physical sciences and it is an open question whether his conjecture would apply to the theory of classical genetics as well. Second, and more importantly, a function or concept that is theoretical for a theory $\mathrm{T}_{1}$ may be non-theoretical for some other theory $\mathrm{T}_{2}$. But that would make it non-causal for $\mathrm{T}_{2}$ by definition. This is an unwelcome consequence of Forge's conjecture.

Second, Kitcher explicitly discusses the notion of causation, but he 'explains it away'. Because of well-known empiricist concerns, he refuses to adopt a causal account of explanation (Kitcher [1989], p. 435). Instead he develops an explanatory account of causation: 'What is distinctive about the unification view is that it proposes to ground causal claims in claims about explanatory dependency rather than vice versa.' (Kitcher [1989], p. 436) Causal relevance is dependent on explanatory relevance, where the latter is tied to the systematization of belief in the limit of scientific inquiry, as guided by the search for derivational unification (Kitcher [1989], p. 499$){ }^{7}$

Third, Darden is less reluctant to talk about causality in her account of classical genetics. She acknowledges that causal considerations did play 
a role in genetics and cytology (Darden [1991], pp. 253-4), especially in the Morgan group (Darden [1991], p. 183), and she uses causal language when formulating the theoretical components of Mendelian genetics (see the examples cited in section 3.3). But for all that, she pays relatively little attention to causation in classical genetics. Nor does she give an account of causation.

One may wonder, of course, whether causality really is that important. ${ }^{8}$ In my opinion, there are multiple reasons why causality merits particular attention in our representations of scientific theories, the most important one being related to explanation. ${ }^{9}$ For many philosophers, explanation is tightly connected to causation; see the causal-mechanical accounts of Salmon ([1984]) and Dowe ([2000]), the interventionist accounts of Hausman ([1998]) and Woodward ([2003]), and the mechanistic accounts of Machamer et al. ([2000]), Glennan ([1996], [2002]), Bechtel and Abrahamsen ([2005]) and Craver ([2007]).

One of the central motivations for stressing the role of causality in explanation is that it solves several of the problems that notoriously plague Hempel's ([1965]) deductive-nomological $(D N)$ model of explanation. (Woodward [2009], section 2.5) One such problem is explanatory asymmetry. One may logically derive, and hence $D N$-explain, the length of a flagpole's shadow from its height, together with certain laws and initial conditions. Likewise, one may derive, and hence $D N$-explain, its height from the length of its shadow, again together with certain laws and initial conditions. But whereas the first derivation seems explanatory, the second does not. Kitcher ([1989], pp. 485-7) endeavours to solve this problem within his unificationist model, but he arguably fails (Woodward [2003], pp. 358-60; Gijsbers [2007], pp. 489-91). Causal considerations, by 
contrast, fit this intuition well: the length of the shadow is caused by the length of the flagpole, but not vice versa.

Before turning to the next section, I would like to add one remark on the relation between explanation and causality. Even though I endorse the view that causality plays an important role in explanation, I do not wish to say that all explanation is causal explanation. Mathematical explanations, for example, may well be a-causal (see Mancosu [2011], section 1 and Woodward [2003], pp. 220-1) and they may better fit Hempel's or Kitcher's account of explanation. That need not be a problem; after all it seems doubtful that there can be a truly general account of explanation that holds in all possible domains of inquiry (cf. note 25). Still, if causal considerations are relevant, they should better be taken into account. This surely holds in the case of classical genetics, as I will argue in the next section.

\section{Woodward's Interventionist Account of Causation}

My account of the causal structure of classical genetics builds upon Woodward's interventionist theory of causation. The central idea of this theory is that causal relations 'are potentially exploitable for purposes of manipulation and control' (Woodward [2003], p. v). For two reasons, Woodward's theory is well suited for my purposes. ${ }^{10}$ First, it dovetails with (large part of) the literature on causal Bayes nets, such as the works of Pearl ([2000]) and Spirtes et al. ([2000]) (see Woodward [2003], pp. 38-45). Second, it also nicely fits the concept of causality as it figured in classical genetics. As Waters ([2007], section I) points out, classical geneticists considered genes as difference makers for phenotypic traits in the way specified by the difference principle (see Waters [1994], p. 172 for a 
seminal version of this principle):

Difference principle: differences in a gene cause uniform phenotypic differences in particular genetic and environmental contexts. (Waters [2007], p. 558)

In well-devised experiments, these classical geneticists created a stable genetic and environmental context so as to make sure that the difference principle would apply (Waters [2007], p. 558). This principle, Waters contends, can be naturally reformulated in terms of manipulations of genes (in the sense of the 'ideal interventions' to be discussed below) given certain genetic and environmental contexts (Waters [2007], p. 564).

Woodward ([2003], p. 39) conceives of the relata of causal relations as random variables (more precisely: changes in the values thereof).

Intuitively, a random variable represents some feature of an entity or set of entities. Each random variable can have several possible values, each of which represents a different state that the feature can take. For example, the variable 'TrafficLights' can take 'red', 'orange' and 'green' as its possible values.

The basic intuition underlying Woodward's account of causation is that one random variable $X$ is a (type-level) cause of another random variable $Y$ just in case manipulating $X$ would result in a change in $Y$ (or in the probability distribution of $Y$ ). Of course, not every manipulation of $X$ would be suitable. If, for example, the manipulation of $X$ would change $Y$ directly, or if it would change some of the causes of $Y$ that are not themselves effects of $X$, then $Y$ would change irrespective of whether $X$ is a cause of $Y$. To rule out such problems, Woodward introduces the notion of an ideal intervention on $X$ with respect to $Y$ to explicate changes in $X$ 
which influence $Y$, if at all, only via $X$ and not via some other causal route (see Woodward [2003], p. 94 for an informal characterization and 98-99 for a precise definition). If the relation between $X$ and $Y$ remains 'invariant', that is, continues to hold, under some range of such ideal interventions on $X$, then it is causal. It is not necessary that it is invariant under all possible interventions on $X$; 'invariance under interventions' is a gradual notion (Woodward [2003], pp. 257-65). With the help of the notion of ideal interventions, Woodward defines 'direct causation' (relative to a set of variables $V$ ) as follows (this notion nicely fits the formal framework of causal Bayes nets.):

(DC) A necessary and sufficient condition of $X$ to be a direct cause of $Y$ with respect to some variable set $V$ is that there be a possible intervention on $X$ that will change $Y$ (or the probability distribution of $Y$ ) when all other variables in $V$ besides $X$ and $Y$ are held fixed at some value by interventions. (Woodward [2003], p. 55)

\section{Causal Bayes Nets and Their Interrelations}

In this section, I first introduce the basic terminology of causal Bayes nets (6.1). Then I introduce some new concepts: various isomorphism relations for causal nets and their constituents (6.2). Finally, I discuss a related concept - credal nets - and define corresponding isomorphism relations (6.3). Causal nets will play the same role as models in the structuralist framework. Credal nets will serve in the creation of a causal analogue for structuralist theory-elements and Darden's abstract explanatory patterns. And the isomorphism relations will help to explore classical genetics' unifying power. 


\subsection{Causal Bayes nets}

A causal net (or causal Bayesian network) is a causally interpreted Bayesian network. A Bayesian network $\mathcal{B}=\langle G, P\rangle$ consists of a directed acyclic graph $G=\langle V, E\rangle$ and a probability distribution $P(V)$ over a set of random variables $V$, where $P(V)$ and $G$ satisfy the Markov Condition. (If it is clear over which set of variables a distribution is defined, I will write $P$ instead of $P(V)$.) A directed acyclic graph $G=\langle V, E\rangle$ consists of a set $V$ of vertices or nodes (this is the set of random variables on which $\mathcal{B}$ is defined), and a set $E$ of directed edges $(A \rightarrow B$, where $A, B \in V)$. Since $G$ is acyclic, there is no directed path from any variable to itself.

To interpret a Bayesian network causally (and hence to treat it as a causal net) is to interpret the edges in $E$ causally, so that for all vertices $A, B \in V, A \rightarrow B \in E$ if and only if $A$ is a direct cause of $B$, relative to $V$. The notion of 'direct cause' can be defined by means of Woodward's (DC).

In the case of causal nets, the Markov Condition is called the Causal Markov Condition. Let $D E S C(A)$ consist of all variables $B \in V$ such that in $G$ there is a directed path from $A$ to $B$ (these are the graph-theoretical 'descendants' of $A$ ); and let $P A(A)$ consist of all $C \in V$ such that $C \rightarrow A \in E$ (these are the graph-theoretical 'parents' of $A$ ).

Definition 1 (Causal Markov Condition) Let $G=\langle V, E\rangle$ be a causal graph with vertex set $V$ and $P$ be a probability distribution over the vertices in $V$ generated by the causal structure represented by $G$. $G$ and $P$ satisfy the Causal Markov Condition if and only if for every $A \in V, A$ is independent of $V \backslash(D E S C(A) \cup P A(A))$ given $P A(A) .{ }^{11}$ (Adapted from Spirtes et al. [2000], p. 29)

The Causal Markov Condition specifies which conditional or 
unconditional independence relations a probability distribution $P$ must satisfy in order to be causally Markov with respect to a given graph $G$, so that $\mathcal{B}=\langle G, P\rangle$ counts as a causal net. ${ }^{12}$ This can be illustrated by means of figure 1. $P T_{1}$ is independent of $G C_{1}$ conditional on $G T_{1}$ in any $P$ that is causally Markov to figure 1; likewise, $G C_{1}$ and $G C_{2}$ are unconditionally independent. (At the moment, the reader need not care about what these variables stand for or which causal relations this figure depicts.)

\subsection{Relations among causal nets}

As is evident from the above definitions, causal nets may differ from each other along several lines: viz. with respect to $V$ and/or $E$ and/or $P$. Here I will define several possible relations between sets of variables, between graphs, and between causal nets that are helpful to analyse the causal structure of classical genetics.

But first let me introduce some notation pertaining to random variables. Each random variable $A \in V$ may assume a range of possible values. These are mutually exclusive and jointly exhaustive. Let $[A]$ be the set of $A$ 's values. In this paper, I will only deal with finite random variables (variables with a finite number of values). Where $U=\left\{A_{1}, \ldots, A_{n}\right\}$ is a set of variables, let $[U]=\left[A_{1}\right] \times \ldots \times\left[A_{n}\right]$. The members of $[U]$ thus consist of the possible configurations of members of $\left[A_{1}\right], \ldots,\left[A_{n}\right]$, respectively. ${ }^{13}$ Finally, let $[[U]]=\bigcup\left[A_{i}\right]$ (for all $\left.A_{i} \in U\right)$. Obviously, $[U]$ and $[[U]]$ are different sets.

Now we are ready to define some possible interrelations between causal nets. I will start with isomorphism and value-isomorphism, defining these notions in turn for sets of variables, for graphs, and for causal nets.

Definition 2 ((value-)isomorphism for sets of variables) Two sets 
of variables $V$ and $V^{\prime}$ are isomorphic if and only if there is a bijection $b: V \rightarrow V^{\prime}{ }^{14}$ They are value-isomorphic if and only if there are bijections $b: V \rightarrow V^{\prime}$ and $b^{\prime}:[[V]] \rightarrow\left[\left[V^{\prime}\right]\right]$ such that for any $A \in V$ and $a \in[[V]]$, $a \in[A]$ if and only if $b^{\prime}(a) \in[b(A)]$.

Definition 3 ((value-)isomorphism for graphs) Two graphs $G=\langle V, E\rangle$ and $G^{\prime}=\left\langle V^{\prime}, E^{\prime}\right\rangle$ are isomorphic if and only if there is a bijection $b: V \rightarrow V^{\prime}$ such that for any $A, B \in V: A \rightarrow B \in E$ if and only if $b(A) \rightarrow b(B) \in E^{\prime}$. They are value-isomorphic if and only if they are isomorphic and $V$ and $V^{\prime}$ are value-isomorphic.

Definition 4 ((value-)isomorphism for causal nets) Two causal nets $\mathcal{B}=\langle G, P\rangle$ and $\mathcal{B}^{\prime}=\left\langle G^{\prime}, P^{\prime}\right\rangle$ are isomorphic if and only if $G$ and $G^{\prime}$ are isomorphic. They are value-isomorphic if and only if $G$ and $G^{\prime}$ are value-isomorphic.

With respect to isomorphic graphs and causal nets, let me introduce the following convention:

Convention 1 If $G=\langle V, E\rangle$ and $G^{\prime}=\left\langle V^{\prime}, E^{\prime}\right\rangle$ are isomorphic, I will write $G^{\prime}=\left\langle V^{\prime}, E\right\rangle$ instead of $G^{\prime}=\left\langle V^{\prime}, E^{\prime}\right\rangle$-even if $E$ and $E^{\prime}$ are specified over different sets of variables. Analogously, I will write $\mathcal{B}^{\prime}=\left\langle\left\langle V^{\prime}, E\right\rangle, P^{\prime}\right\rangle$ instead of $\mathcal{B}^{\prime}=\left\langle\left\langle V^{\prime}, E^{\prime}\right\rangle, P^{\prime}\right\rangle$ in case $\mathcal{B}$ and $\mathcal{B}^{\prime}$ are isomorphic.

The relations of isomorphism and value-isomorphism of definition 4 concern structural (or qualitative) similarities between causal nets. Let us now turn to probabilistic (or quantitative) similarities.

Definition 5 (distribution-identity for causal nets) Let $\mathcal{B}=\langle\langle V, E\rangle$, $P\rangle$ and $\mathcal{B}^{\prime}=\left\langle\left\langle V^{\prime}, E\right\rangle, P^{\prime}\right\rangle$ be value-isomorphic and let $b$ and $b^{\prime}$ be bijections as in definition 2. Moreover, for every $u=\left\langle a_{1}, a_{2}, \ldots, a_{n}\right\rangle \in[V]$, let 
$b^{\prime}(u)=\left\langle b^{\prime}\left(a_{1}\right), b^{\prime}\left(a_{2}\right), \ldots, b^{\prime}\left(a_{n}\right)\right\rangle$. Then $\mathcal{B}$ and $\mathcal{B}^{\prime}$ are distribution-identical if and only if $P(u)=P^{\prime}\left(b^{\prime}(u)\right)$ for each $u \in[V]$. (In other words, $\mathcal{B}$ and $\mathcal{B}^{\prime}$ have like joint distributions.)

The relations between these definitions are obvious. If two sets of variables $V$ and $V^{\prime}$ are value-isomorphic they are also isomorphic, but not vice versa. (Idem for graphs and causal nets.) If two causal nets $\mathcal{B}$ and $\mathcal{B}^{\prime}$ are isomorphic (resp. value-isomorphic), then so are their respective graphs and hence their respective sets of variables, but not vice versa. And if two causal nets are distribution-identical they are value-isomorphic, but not vice versa.

\subsection{Credal nets and their interrelations}

Along with causal nets, I will use another concept that ties graphs and probability distributions together: credal nets. A credal net is a set of Bayesian networks over a fixed set of variables (Cozman [2005], p. 171). ${ }^{15}$ These Bayes nets all have the same $G$, but differ with respect to $P$. More precisely, a credal net $\mathfrak{B}=\langle G, \mathbb{P}\rangle$ consists of a directed acyclic graph $G=\langle V, E\rangle$ and a set $\mathbb{P}$ of probability distributions over $V$, called a a credal set. As I interpret Bayes nets causally here, I will carry over this interpretation to credal nets.

Definition 6 (credal net) A credal net is a set of causal nets with a common graph: $\mathfrak{B}=\langle G, \mathbb{P}\rangle=\{\mathcal{B}=\langle G, P\rangle \mid P \in \mathbb{P}\}$.

Structural (qualitative) and probabilistic (quantitative) similarity relations can also be defined for credal sets and credal nets: 
Definition 7 ((value-)isomorphism for credal sets) Two credal sets $\mathbb{P}(V)$ and $\mathbb{P}^{\prime}\left(V^{\prime}\right)$ are isomorphic if and only if $V$ and $V^{\prime}$ are isomorphic. They are value-isomorphic if and only if $V$ and $V^{\prime}$ are value-isomorphic.

Definition 8 (distribution-identity for credal sets) Let $V$ and $V^{\prime}$ be value-isomorphic, let $b$ and $b^{\prime}$ be bijections as in definition 2 , and let $u$ and $b^{\prime}(u)$ be defined as in definition 5. Then the credal sets $\mathbb{P}(V)$ and $\mathbb{P}^{\prime}\left(V^{\prime}\right)$ are distribution-identical if and only if there is a bijection $b^{\prime \prime}: \mathbb{P} \rightarrow \mathbb{P}^{\prime}$ such that $P(u)=b^{\prime \prime}(P)\left(b^{\prime}(u)\right)$ for all $P \in \mathbb{P}$ and all $u \in[V]$. (In other words, all distributions in $\mathbb{P}$ are distribution-identical to their image in $\mathbb{P}^{\prime}$.)

Definition 9 ((value-)isomorphism for credal nets) Two credal nets $\mathfrak{B}=\langle G, \mathbb{P}\rangle$ and $\mathfrak{B}^{\prime}=\left\langle G^{\prime}, \mathbb{P}^{\prime}\right\rangle$ are isomorphic if and only if $G$ and $G^{\prime}$ are isomorphic. They are value-isomorphic if and only if $G$ and $G^{\prime}$ are value-isomorphic.

Definition 10 (distribution-identity for credal nets) Two credal nets $\mathfrak{B}=\langle\langle V, E\rangle, \mathbb{P}\rangle$ and $\mathfrak{B}^{\prime}=\left\langle\left\langle V^{\prime}, E\right\rangle, \mathbb{P}^{\prime}\right\rangle$ are distribution-identical if and only if $\mathbb{P}(V)$ and $\mathbb{P}^{\prime}\left(V^{\prime}\right)$ are distribution-identical. Equivalently, $\mathfrak{B}$ and $\mathfrak{B}^{\prime}$ are distribution-identical if and only if there is a bijection $b^{*}: \mathfrak{B} \rightarrow \mathfrak{B}^{\prime}$ such that for all $\mathcal{B} \in \mathfrak{B}, \mathcal{B}$ is distribution-identical to $b^{*}(\mathcal{B})$.

The relations between these definitions are obvious. If two credal sets $\mathbb{P}$ and $\mathbb{P}^{\prime}$ are distribution-identical they are also value-isomorphic, and hence also isomorphic, but not vice versa. (Idem for credal nets.) And if two credal nets $\mathfrak{B}$ and $\mathfrak{B}^{\prime}$ are distribution-identical, then so are their respective credal sets. (Idem for value-isomorphism and isomorphism.)

Overview of the above definitions: 


\begin{tabular}{|l|l|l|}
\hline & (value-)isomorphism & distribution-identity \\
\hline sets of variables & definition 2 & $/$ \\
graphs & definition 3 & $/$ \\
causal nets & definition 4 & definition 5 \\
credal sets & definition 7 & definition 8 \\
credal nets & definition 9 & definition 10 \\
\hline
\end{tabular}

\section{The Theory of the Gene and its Causal Graph}

Let us turn now to the second part of this paper and apply the above formal framework to Morgan's exposition of the 'theory of the gene' (Morgan [1928]). According to Morgan, the modern theory of heredity 'is primarily concerned with the distribution of units between successive generations of individuals' (Morgan [1928], p. 1). These units are invisible and they are called genes. To these genes, properties are assigned in a non-arbitrary way, based on 'numerical data obtained by crossing two individuals that differ in one or more characters' (Morgan [1928], p. 1). After presenting several examples of possible relations between genes and characters, Morgan formulates the theory of the gene as follows (the three following quotes are from Morgan [1928], p. 25):

We are now in a position to formulate the theory of the gene.

The theory states that the characters of the individual are referable to paired elements (genes) in the germinal material that are held together in a definite number of linkage groups;

Where Morgan writes about 'pairs of genes', we would now say 'pairs of alleles.' Linkage groups are groups of genes that are typically transmitted together (since they lie on the same chromosome). Morgan continues by 
specifying the law of segregation (Mendel's first law) and the law of independent assortment (Mendel's second law):

it states that the members of each pair of genes separate when the germ-cells mature in accordance with Mendel's first law, and in consequence each germ-cell comes to contain one set only; it states that the members belonging to different linkage groups assort independently in accordance with Mendel's second law;

Finally he explains the basics of 'gene mapping', the technique by means of which the Morgan group was able to discover which of an organism's genes belonged to the same linkage group (and in what order):

it states that an orderly interchange - crossing-over - also takes place, at times, between the elements in corresponding linkage groups; and it states that the frequency of crossing-over furnishes evidence of the linear order of the elements in each linkage group and of the relative position of the elements with respect to each other.

The theory of the gene, as it is presented by Morgan, is not one single theory. It is composed of different abstract explanatory patterns (Darden [1991]), general argument patterns (Kitcher [1989]), or theory-elements forming a theory-net (Balzer and Lorenzano [2000]). I will incorporate this aspect by representing it by means of interrelated credal nets. As these credal nets are interpreted causally, the common lacuna is remedied. To some extent these credal nets share the same causal structure. This common causal structure ties them together, and distinguishes the theory 


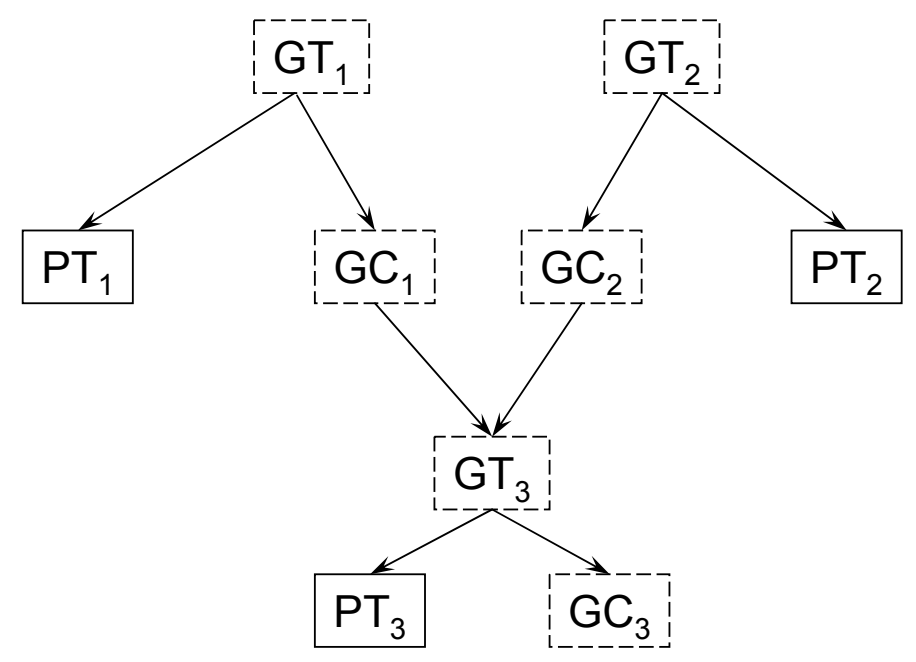

Figure 1: The causal structure of classical genetics

of the gene from alternative theories of inheritance such as Francis Galton's (see Leuridan [2007] for a brief characterization of Galton's theory).

This shared causal structure is shown in figure 1. The directed acyclic graph comprises nine nodes, which represent the genotype $\left(G T_{i}\right)$, the phenotype $\left(P T_{i}\right)$ and the make-up of the germ-cells $\left(G C_{i}\right)$ of three groups of organisms (a paternal group, $i=1$; a maternal group, $i=2$; and a filial group, $i=3) .{ }^{16}$ The $G T_{i}$ and $G C_{i}$ variables in figure 1 are dotted to indicate that they are CG-theoretical in the structuralists' sense (see section 3.1 ; 'CG' stands for the theory of classical genetics). At the time, there was no way to determine an organism's genotype or the make-up of its germ-cells without invoking the theory of classical genetics. The phenotypic variables, by contrast, are CG-non-theoretical. One can determine an organism's phenotypic traits without relying on CG. This point is important with respect to explanation in classical genetics (see section 17). 
The causal relations between these variables are depicted in figure 1 . The genotypes $\left(G T_{i}\right)$ causally influence the corresponding phenotypes $\left(P T_{i}\right)$, as well as the corresponding germ cells $\left(G C_{i}\right)$-manipulating the former via an ideal intervention will change (the probability distribution over) the latter. And the parental germ cells $\left(G C_{1}\right.$ and $\left.G C_{2}\right)$ together causally determine the filial genotype $G T_{3}$. These relations are very plausible from a present-day point of view. But to avoid any allegation that I'm misinterpreting Morgan's exposition, we should briefly justify them on the basis of his work.

In section 5, I have argued, based on (Waters [2007]), that Woodward's interventionism fits Morgan's concept of causality. Here I discuss the precise causal relations that played a role in Morgan's theory of the gene. Let me start with the relation between genes and characters. It is very natural to consider genes as the causes of characters; i.e. to assume that for each $i, G T_{i} \rightarrow P T_{i}$. It is not so natural, however, to attribute this assumption to Morgan and his contemporaries. Explicit causal language was surprisingly rare in the genetics literature at the time. Still, Morgan uses implicit causal language on repeated occasions. He hypothesizes that the tall variety of pea plants 'contains in its germ-cells something that makes the plants tall' (Morgan [1928], p. 2, my emphasis). He distinguishes between red-producing genes and white-producing genes in crosses of four-o'clocks (Morgan [1928], p. 8). And he talks about the effects of mutant changes (Morgan [1928], p. 315). These examples should suffice to show that, according to Morgan, genes cause characters (see also Darden [1991], pp. 182-3 and Waters [2007]).

What about the relations between the paired genes and the germcells? In the third and the fourth chapter of his book, Morgan lists overwhelming 
cytological evidence leading to the conclusion that $G T_{i} \rightarrow G C_{i}(1 \leq i \leq 3)$ and that $G C_{1} \rightarrow C T_{3} \leftarrow G C_{2}$ : the chromosomes are the bearers of the genes ([1928], p. 45), somatic cells have a diploid number of chromosomes, half of which come from the father, the other half from the mother ([1928], p. 32), and the germ cells receive a haploid number of chromosomes ([1928], p. 33).

\section{A First Exemplar: Stem Length in Pea Plants}

Morgan ([1928]) presents the theory of classical genetics by means of a set of exemplars, particular crosses which are explained by means of the theory of the gene and which are supposed to represent a class of similar phenomena. His first exemplar consists of Mendel's well-known monohybrid crosses with complete dominance on pea plants (Morgan [1928], pp. 2-4). Recall that in a monohybrid cross only one pair of opposing characteristics is studied.

\subsection{Three crosses on stem length in pea plants}

Mendel ([1865], pp. 5-27) crossed a tall variety of edible pea (Pisum) with a short variety. The tall plants he used were true-breeding. He observed that when true-breeding tall plants are crossed with short plants, all the offspring or hybrids $\left(F_{1}\right)$ are tall (cross 1$)$. It did not matter whether the tall plants produced pollen and the short plants produced eggs or vice versa. Reciprocal crosses gave identical results (Mendel [1865], pp. 8-9). In a second cross (cross 2), self-fertilization of the $F_{1}$-generation resulted in offspring $\left(F_{2}\right) 75 \%$ of which was tall, the other $25 \%$ being short. Finally, when the $F_{1}$ hybrids (pollen plants) were back-crossed to short plants (egg plants), $50 \%$ of the resulting off-spring $F_{2^{\prime}}$ was tall, the other $50 \%$ was 
short (cross 3). How can these phenotypic distributions be explained? It will turn out in section 9 that the results of each cross can be explained by fitting them in a causal net the graph of which is isomorphic to figure 1. Here, I will first characterize the graph which these causal nets have in common and the explanatory principles or laws their probability distributions have to satisfy.

\subsection{The causal graph for stem length in pea plants}

Let us first give an appropriate interpretation for the nodes in figure 1 by specifying their respective sets of possible values. All the plants in these crosses are tall $\left(P T_{i}=\right.$ tall $)$ or short $\left(P T_{i}=\right.$ short $)$. These traits are referable to paired alleles in the germinal material. Let $t$ and $s$ denote a 'tall-producing' and a 'short-producing' allele, respectively. ${ }^{17}$ There are three possible configurations of paired alleles, i.e. three possible values for the variables $G T_{i}$, viz. $t t, t s$, and $s s$. Finally, the germ-cells contain one allele of each pair. Hence, the variables $G C_{i}$ may assume the values $t$ or $s$.

These specifications can be summarized as follows. Let $V^{1}=\left\{P T_{1}, \ldots, G C_{3}\right\}$ be a set of variables corresponding to the nodes in figure 1. Let $\left[P T_{i}\right]=\{$ tall, short $\},\left[G T_{i}\right]=\{t t, t s, s s\}$, and $\left[G C_{i}\right]=\{t, s\},(1 \leq i \leq 3)$. Let $G^{1}=\left\langle V^{1}, E\right\rangle$ be isomorphic to figure 1 (where $P T_{1} \in V^{1}$ corresponds to the node $P T_{1}$ in the graph, ...). This completes the description of $G^{1}$. By adding probability distributions to this graph, we will obtain causal nets that represent crosses 1 to 3 . These probability distributions have to satisfy the explanatory principles, or laws, invoked by Morgan. 


\begin{tabular}{|l|ll|}
\multicolumn{2}{c}{ table 1} \\
\hline \multirow{3}{*}{ GT } & \multicolumn{2}{|c|}{$P T_{i}$} \\
tall & short \\
\hline$t t$ & 1.00 & 0.00 \\
$t s$ & 1.00 & 0.00 \\
$s s$ & 0.00 & 1.00 \\
& & \\
\hline
\end{tabular}

\begin{tabular}{|l|cc|}
\multicolumn{3}{c}{ table 2} \\
\hline$G T_{i}$ & \multicolumn{2}{|c|}{$G C_{i}$} \\
\hline$t t$ & 1.00 & 0.00 \\
$t s$ & 0.50 & 0.50 \\
$s s$ & 0.00 & 1.00 \\
\hline
\end{tabular}

\begin{tabular}{|ll|ccc|}
\multicolumn{5}{|c|}{ table 3} \\
$G C_{1}$ & $G C_{2}$ & $t t$ & $t s$ & $s s$ \\
\hline$t$ & $t$ & 1.00 & 0.00 & 0.00 \\
$t$ & $s$ & 0.00 & 1.00 & 0.00 \\
$s$ & $t$ & 0.00 & 1.00 & 0.00 \\
$s$ & $s$ & 0.00 & 0.00 & 1.00 \\
\hline
\end{tabular}

Conditional probability tables $1-3$, satisfied by all $P \in \mathbb{P}^{1}$

\subsection{Morgan's explanatory principles and the credal net for stem length in pea plants}

To explain the phenotypic distributions of crosses 1 to 3, Morgan appeals to four principles - either explicitly or implicitly ([1928], pp. 2-4). First, he assumes that plants having two tall-producing alleles are tall; that hybrids, which have an allele for tall and one for short, are tall; and that plants having two short-producing alleles are short. This corresponds to what is called the principle of complete dominance. They are summarized in conditional probability table 1 . This table specifies the probability distribution over the values of each $P T_{i}$, given the possible values of its graphical parent $G T_{i},(1 \leq i \leq 3)$. For example, the second row states that $P\left(P T_{i}=\right.$ tall $\left.\mid G T_{i}=t s\right)=1.00$. This expresses the dominance of tall over short.

Second, Morgan invokes the law of segregation (Mendel's first law): '[i]f the element [or allele] for tall and the one for short (that are both present in the hybrid) separate in the hybrid when the eggs and pollen grains come to maturity, half the eggs will contain the tall and half the short element [...]. Similarly for the pollen grains' (Morgan [1928], p. 3). In this quote, Morgan only discusses segregation for hybrid plants, but from his exposition of the underlying chromosomal mechanism, it is clear that Mendel's first law applies to true-breeding plants as well, see (Morgan 
[1928], pp. 33-4). The law of segregation is summarized in table 2.

A third, very implicit principle states that when an egg and a pollen grain fertilize, their respective elements together make up the genotype of the resulting offspring. I will call this the combination principle (table 3$).{ }^{18}$

Finally, Morgan assumes chance fertilization ([1928], p. 3). For example, when one crosses or self-fertilizes hybrids it may not be the case that tall-producing pollen have a tendency to fertilize tall-producing eggs rather than short-producing eggs. This assumption need not be expressed by means of a conditional probability table, as it holds for any probability distribution that satisfies the Causal Markov Condition relative to figure $1 .{ }^{19}$

Together, these principles give rise to a credal set $\mathbb{P}^{1}\left(V^{1}\right)$ :

$$
\begin{aligned}
\mathbb{P}^{1}\left(V^{1}\right)= & \left\{P\left(V^{1}\right) \mid P\left(V^{1}\right) \text { satisfies tables } 1-3\right. \text { and } \\
& \text { the Causal Markov Condition relative to } \left.G^{1}\right\} .
\end{aligned}
$$

Joined with $G^{1}$, this defines a credal net $\mathfrak{B}^{1}=\left\langle G^{1}, \mathbb{P}^{1}\right\rangle$.

\section{Explaining Mendel's Crosses: a Causal-Structural Account}

Morgan explains the filial phenotypic distributions in crosses 1 to 3 by deriving them, by means of the laws or explanatory principles just discussed, from assumptions regarding the parental genotypes. Described in this way, explanation is syntactic. But we can also approach it in a semantic or model-theoretic way. Each of the crosses can be explained by embedding them in a causal net (some $\mathcal{B} \in \mathfrak{B}^{1}$ ). This model-theoretic approach mimics the structuralist account of explanation, while making room for the concept of causality. Let me call it the causal-structural 
account of explanation. (See section 17 for a further elaboration.)

In section 3.1 we saw that for the structuralists, to explain some given data by means of a theory $\mathbf{T},{ }^{20}$ one adds to these data - which have the structure of a partial potential model-T's theoretical concepts - thus creating a potential model - and then shows that it is an actual model of T. This 'embedding' can be translated to the language of causal nets. ${ }^{21}$

The data in section 8.1 concern the phenotypic distribution of the filial group, given the phenotypes of the parental groups. Hence they pertain to the CG-non-theoretic variables $P T_{i}$ (with $\left[P T_{i}\right]=\{$ tall, short $\}, 1 \leq i \leq 3$ ). These can be included in a graph that also contains the CG-theoretical variables $G T_{i}$ and $G C_{i}(1 \leq i \leq 3)$. By demanding that this graph is isomorphic to figure 1 and by adding suitable probability distributions which satisfy tables 1-3 and are causally Markov to the graph in question, one obtains models (causal nets in $\mathfrak{B}^{1}$ ) that incorporate classical genetics' conceptual framework, satisfy its laws and explain the data.

Let us first turn to cross 1 . In one variant of cross 1 , the pollen plants are true-breeding for tall and the egg plants are short: $P T_{1}=$ tall, $P T_{2}=$ short . If it is assumed that a true-breeding tall plant contains the element for tall twice (cf. Morgan [1928], p. 6), it can be 'abduced' that $G T_{1}=t t$ and $G T_{2}=s s .{ }^{22}$ If we denote the probability distribution corresponding to this cross by $P^{1}\left(V^{1}\right)$, or briefly $P^{1}$, we can express this as follows: $P^{1}\left(P T_{1}=\right.$ tall $)=P^{1}\left(P T_{2}=\right.$ short $)=$ 1.00 , and $P^{1}\left(G T_{1}=t t\right)=P^{1}\left(G T_{2}=s s\right)=1.00$. Assume now that $P^{1} \in \mathbb{P}^{1}$, so that it satisfies tables $1-3$ and the corresponding explanatory principles. Then it follows that $P^{1}\left(G C_{1}=t\right)=P^{1}\left(G C_{2}=s\right)=1.00$ (by table 2 ), and that $P^{1}\left(G T_{3}=t s\right)=1.00$ (by table 3 ). Hence $P^{1}\left(P T_{3}=\right.$ tall $)=1.00$ (by table 1$)$, which corresponds to the data. This 
shows that cross 1 can be represented by a causal net $\mathcal{B}^{1}=\left\langle G^{1}, P^{1}\right\rangle$ which predicts that all hybrid offspring is tall. Since $\mathcal{B}^{1}$ satisfies causal explanatory principles, this embedding of the data in $\mathcal{B}^{1}$ can rightly be called causal explanation. It shows how the variables on which the data report (in casu $P T_{3}$ ) fit in a larger causal system, and how this causal system brings about the (probability distribution over the) values of these variables. $^{23}$

The phenotypic distribution in cross 2 can be explained in an analogous way, by embedding it in some causal net, say $\mathcal{B}^{2}=\left\langle G^{1}, P^{2}\right\rangle$, where $P^{2} \in \mathbb{P}^{1}$ so that $\mathcal{B}^{2} \in \mathfrak{B}^{1}$. In cross 2 , the $F_{1}$ hybrids are selfed and they produce $F_{2}$ offspring $75 \%$ of which is tall and $25 \%$ of which is short. Obviously, if $P^{2}\left(G T_{1}=t s\right)=P^{2}\left(G T_{2}=t s\right)=1.00$ and if $P^{2} \in \mathbb{P}^{1}$, then $P^{2}\left(G T_{3}=t t\right)=0.25, P^{2}\left(G T_{3}=t s\right)=0.50$, and $P^{2}\left(G T_{3}=s s\right)=0.25$, so that $P^{2}\left(P T_{3}=\right.$ tall $)=0.75$ and $P^{2}\left(P T_{3}=\right.$ short $)=0.25$. This corresponds to the data.

Finally, in cross 3 the $F_{1}$ hybrids are back-crossed with the recessive parental plants. Let $P^{3}\left(G T_{1}=t s\right)=P^{3}\left(G T_{2}=s s\right)=1.00$. If $P^{3} \in \mathbb{P}^{1}$, then $P^{3}\left(P T_{3}=\right.$ tall $)=0.50$. 'The results confirm the expectation.' (Morgan [1928], p. 4) Hence, cross 3 can be represented by $\mathcal{B}^{3}=\left\langle G^{1}, P^{3}\right\rangle \in \mathfrak{B}^{1}$.

Cross 3 was designed by Mendel (and cited by Morgan) as a test cross. The fact that several different, but somehow similar crosses could be explained by means of the same explanatory principles, raised confidence that the explanatory principles invoked were not ad hoc.

So far we have seen that a number of crosses with tall and short pea plants can be represented by distinct causal nets $\mathcal{B}^{1}, \mathcal{B}^{2}, \mathcal{B}^{3}$ that belong to one common credal net $\mathfrak{B}^{1}$. Each of these causal nets is a model of 
classical genetics and explains the data (phenotypic distributions) at hand. In what follows, I will show how crosses on other characters in Pisum or with other genera can be represented by causal nets that fit in analogous credal nets, and explicate the precise relations between these credal nets. (This will prove to be fruitful when we turn to the discussion of explanatory unification.)

\section{Monohybrid Crosses with Complete Dominance}

$\mathfrak{B}^{1}$ 's can be used to describe monohybrid crosses with tall (dominant) and short (recessive) Pisum plants. In the history of classical genetics, many other monohybrid crosses with complete dominance have been reported. In his Versuche über Pflanzen-Hybriden, Mendel discussed six more such crosses with Pisum (Mendel [1865], pp. 5-17). He selected traits relating to the shape of the ripe seeds (round, wrinkled), the colouration of the seed albumen (yellow, green), ... Likewise, Morgan ([1928], pp. 4-5) discusses the inheritance of eye colour in humans (blue, brown) as an example of complete dominance. These crosses cannot be described by the members of $\mathfrak{B}^{1}$, since the set of variables $V^{1}$ on which these are defined is tied to stem length in Pisum. However, by slightly changing the members in $V^{1}$, they can.

Consider Morgan's case of human eye colour. Two phenotypic traits are studied, where brown is dominant to blue. These are referable to paired alleles in the germinal material, which Morgan denotes by $b r$ and $b l$ respectively. (Morgan and his co-workers used a large variety of symbolic systems to denote alleles.) The results of crosses with blue and brown eyes in humans can be accounted for with the help of the explanatory principles discussed in section 8.3, viz. the law of segregation, complete dominance, 
etc. Thus, they are very similar to the case of stem length in Pisum. This similarity can be explicated in a precise way. There is a credal net, say $\mathfrak{B}^{2}$, that is both value-isomorphic and distribution-identical to $\mathfrak{B}^{1}$, such that its members can be used to explain crosses on eye colour in humans.

Let us see how $\mathfrak{B}^{2}$ can be characterized. $V^{2}=\left\{P T_{1}^{\prime}, \ldots, \ldots, G C_{3}^{\prime}\right\}$ is a set of variables corresponding to the nodes in figure 1, where $\left[P T_{i}^{\prime}\right]=\{$ brown, blue $\},\left[G T_{i}^{\prime}\right]=\{b r b r, b r b l, b l b l\}$, and $\left[G C_{i}^{\prime}\right]=\{b r, b l\},(1 \leq i \leq 3)$. Let $b: V^{1} \rightarrow V^{2}$ be a bijection such that $b\left(P T_{i}\right)=P T_{i}^{\prime}, b\left(G T_{i}\right)=G T_{i}^{\prime}, b\left(G C_{i}\right)=G C_{i}^{\prime}(1 \leq i \leq 3)$. Let $b^{\prime}:\left[\left[V^{1}\right]\right] \rightarrow\left[\left[V^{2}\right]\right]$ be a bijection such that for any $A \in V^{1}$ and $a \in\left[\left[V^{1}\right]\right]$, $a \in[A]$ if and only if $b^{\prime}(a) \in[b(A)]$. More specifically, let $b^{\prime}($ tall $)=$ brown $, b^{\prime}($ short $)=b l u e, b^{\prime}(t t)=b r b r, \ldots, b^{\prime}(s)=b l . V^{2}$ is value-isomorphic to $V^{1}$ (definition 2); hence $G^{2}=\left\langle V^{2}, E\right\rangle$ is value-isomorphic to $G^{1}=\left\langle V^{1}, E\right\rangle$ (definition 3).

Let $\mathbb{P}^{2}$ be the set of probability distributions over $V^{2}$ that satisfy constraints analogous to tables 1-3 and are causally Markov to $G^{2}$. More specifically, where the members of $\mathbb{P}^{1}$ satisfy $P(a \mid p a(A))=r$ (for some $r \in[0,1])$, let the members of $\mathbb{P}^{2}$ satisfy $P\left(b^{\prime}(a) \mid b^{\prime}(p a(A))\right)=r$ for any $A \in V^{1}, a \in[A]$, and $p a(A) \in[P A(A)]{ }^{24}\left(P A(A) \subset V^{1}\right.$ comprises $A$ 's graphic parents in $G^{1}$.) It follows that $\mathbb{P}^{2}$ is distribution-identical to $\mathbb{P}^{1}$.

Let $\mathfrak{B}^{2}=\left\langle G^{2}, \mathbb{P}^{2}\right\rangle \cdot \mathfrak{B}^{2}$ is value-isomorphic (definition 9) and distribution-identical (definition 10) to $\mathfrak{B}^{1}$. Thus for any possible cross of tall and short pea plants, there is an analogous cross on eye colour in humans. And likewise for their respective explanations.

Formally speaking, it is trivially easy to create a distribution-identical image of a credal net: just relabel the variables and their values. But empirically, the discovery that eye colour could be explained in the very 
same way as stem length, was not trivial at all. Hence distribution-identity expresses a philosophically interesting phenomenon (see also section 18).

\section{Exemplars, Abstract Explanatory Patterns, Generic Credal Nets and Mechanism Schemas}

The relations between crosses on stem length in pea plants and crosses on eye colour in humans can be generalized to all monohybrid crosses with complete dominance. It is useful to introduce the notion of a generic credal net that serves as an abstract representation of all these similar crosses in the same way as do Darden's abstract explanatory patterns. Like abstract explanatory patterns, generic credal nets are obtained by abstracting from exemplars and they play a role in a theory's unifying power.

Darden's notions of 'exemplar' and 'abstract explanatory pattern' derive from the works of Kuhn and Kitcher. Starting from (Kuhn [1962], pp. 186-91), she defines exemplars as '[...] concrete problem solutions in which a formalism (such as a mathematical equation) is applied and given empirical grounding' (Darden [1991], p. 18). Kuhnian exemplars, Darden argues, may serve to generate Kitcherian argument patterns.

[They] may serve in the construction of abstract explanatory patterns or schemas [...]. The patterns abstractly characterize mechanisms, which, when they are operating, produce observable data-points as output. Thus, fitting an observation into a pattern is a way of explaining it. A set of exemplary patterns constitutes the explanatory repertoire of Mendelian genetics [...]. (Darden [1991], p. 19)

The crosses on stem length in Pisum play the role of Dardenian exemplars in (Morgan [1928]). Darden writes, '[t]he examples supplied model cases. 
Similar results of similar hybrid crosses could be explained by invoking similar steps and filling in the details about the characters in the specific cross.' (Darden [1991], p. 18)

Credal nets (such as $\mathfrak{B}^{1}$ and $\mathfrak{B}^{2}$ ) can be looked upon as precise explications of the notion of 'exemplar' in Darden's works. Generic credal nets can be obtained from credal nets by abstracting from the precise details (which genotypes?, which phenotypes?, etc.) of the latter. They are distribution-identical to the credal nets from which they result. Let $\mathfrak{B}^{\alpha}=\left\langle G^{\alpha}, \mathbb{P}^{\alpha}\right\rangle$ be a generic credal net that is distribution-identical to $\mathfrak{B}^{1}$ (with $G^{\alpha}=\left\langle V^{\alpha}, E\right\rangle$ ). Any reciprocal monohybrid cross with two phenotypic traits, two alleles, and complete dominance can be described by means of a causal net that is distribution-identical to some member of $\mathfrak{B}^{\alpha}$, by filling in $V^{\alpha}$ and $\mathbb{P}^{\alpha} \cdot{ }^{25}$

These explications of Darden's concepts are very fruitful, I contend, because they explicitly make room for causality. This fruitfulness will emerge in section 18, where I will provide a causal account of explanatory unification which improves on those of Kitcher and Darden. ${ }^{26}$

Before we go to the next section, Let me briefly turn to Darden's more recent work on mechanisms. Darden now uses the label 'mechanism schema' instead of 'abstract explanatory pattern' ([2002], [2005]). A mechanism schema is a 'truncated abstract description of a mechanism that can be instantiated by filling it with more specific descriptions of component entities and activities' (Darden [2005], pp. 360-1); see also (Machamer et al. [2000], p. 15). ${ }^{27}$ They are often depicted in diagrams (Darden [2005], p. 358). Darden now claims that '[t]he structure of biological theories in [classical genetics and molecular biology] is best analyzed by appeal to mechanism schemas, and not by appeal to sets of 
laws or argument schemata' ([2005], p. 351).

It may be suggested that the notion of 'mechanism schema' provides a more elegant and less formal tool for analysing the theory of classical genetics than mine. ${ }^{28}$ Perhaps, my formal apparatus could be eliminated in favour of the use and reuse with modifications of the diagram in figure 1, interpreted as a mechanism schema. I disagree. Most of Darden's mechanism schemas and the diagrams in which they are depicted, are purely qualitative - as is the graph in figure 1 . But in contrast to the former, the latter may be given a quantitative interpretation in a very natural way, viz. by combining it with causally Markov probability distributions so as to obtain causal nets. (Hence generic credal nets offer an explication of Darden's mechanism schemas; they should not be eliminated in favour of the latter.) As should be clear by now, quantitative information was crucial for the prediction and explanation of phenotypic distributions in classical genetics. In the interest of prediction, biologists like Lazebnik ([2002]) and philosophers like Bechtel ([2011]) have stressed the need for quantitative representations of biologists' findings at the expense of merely qualitative, diagrammatic representations. Casini et al. ([2011]) and Clarke et al. ([unpublished]) have offered accounts of mechanisms invoking causal Bayes nets. These accounts offer an integrated approach to both the qualitative and the quantitative aspects of mechanisms. As a result, one of their major advantages is that they can be easily used for quantitative prediction (both passive prediction and prediction of the results of manipulations). My approach is in line with this interesting work. 


\section{Incomplete Dominance}

$\mathfrak{B}^{1}$ and $\mathfrak{B}^{2}$ represent but two exemplars, and $\mathfrak{B}^{\alpha}$ represents but one part of classical genetics. Obviously there was more to classical genetics than monohybrid crosses with complete dominance. After presenting Mendel's crosses and those regarding eye colour in man, Morgan turns to crosses that are interestingly different and which cannot be represented by means of $\mathfrak{B}^{\alpha}$.

There are other crosses that give, perhaps, a more striking illustration of Mendel's first law. For instance, when a red and a white-flowered four-o'clock [Mirabilis jalapa] are crossed, the hybrid $\left[F_{1}\right]$ has pink flowers [...]. If these pink-flowered hybrid plants self-fertilize, some of their offspring $\left(F_{2}\right)$ are red like one grandparent, some of them pink like the hybrid, and others white like the other grandparent, in the ratio of 1:2:1. Here one original parental color is restored when red germ-cell meets red, the other color is restored when white meets white, and the hybrid combinations appear as often as red meets white, or white meets red. All the colored flowered plants in the second generation taken together are to the white-flowered plants as $3: 1$.

In passing it is important to note two facts. The red and the white $F_{2}$ individuals are expected to breed true, because they contain the elements for red, or for white, twice present [...], but the pink $F_{2}$ individuals should not breed true, since they are like the first hybrid generation, and contain one red and one white element $[\ldots]$. All this turns out to be true when 
these plants are tested. (Morgan [1928], pp. 5-6)

Morgan's explanation of these phenotypic distributions rests on the law of segregation, chance fertilization, and the combination principle. What is new, however, is the relation between the genotype and the phenotype. Let me jointly label the relations in the quote above the 'principle of incomplete dominance'.

How can we account for this difference in terms of causal nets? It is easily seen that crosses with incomplete dominance are structurally different from crosses with complete dominance. Crosses on flower colour in four-o'clocks cannot be described by means of causal nets that are value-isomorphic (let alone distribution-identical) to members of $\mathfrak{B}^{\alpha}$, since the corresponding set of variables, say $V^{3}$, is not value-isomorphic to $V^{\alpha}$. The flower colour of four-o'clocks has three possible states: white, pink, and red. So let me define $V^{3}=\left\{P T_{1}, \ldots, \ldots, G C_{3}\right\}$, with $\left[P T_{i}\right]=\{$ red,pink, white $\},\left[G T_{i}\right]=\{r r, r w, w w\}$, and $\left[G C_{i}\right]=\{r, w\},(1 \leq i \leq 3) .{ }^{29}$ Let $G^{3}=\left\langle V^{3}, E\right\rangle$ be isomorphic to figure 1. What about the probability distributions over $G^{3}$ ? The constraints generated by incomplete dominance, by Mendel's first law, and by the combination principle are summarized in tables 4,5 and 6 , respectively. Together, they determine the credal set $\mathbb{P}^{3}\left(V^{3}\right)$ and the resulting credal net $\mathfrak{B}^{3}=\left\langle G^{3}, \mathbb{P}^{3}\right\rangle$. (As before, chance fertilization is ingrained in the graphical structure of figure 1.)

In line with section 9, the crosses cited by Morgan can be explained by means of members of $\mathfrak{B}^{3}$. In a first cross, a red flowered four-o'clock is joined with a white-flowered four-o'clock. Morgan does not clarify which of the two is the pollen producing plant but, as reciprocal crosses again give the same results, we may assume that the pollen producing plant is red. ${ }^{30}$ 


\begin{tabular}{|l|ccl|}
\multicolumn{5}{c|}{ table 4} \\
\hline$G T_{i}$ & red & pink & white \\
\hline$r r$ & 1.00 & 0.00 & 0.00 \\
$r w$ & 0.00 & 1.00 & 0.00 \\
$w w$ & 0.00 & 0.00 & 1.00 \\
& & & \\
\hline
\end{tabular}

\begin{tabular}{|l|cc|}
\multicolumn{2}{c}{ table 5} \\
\hline$G T_{i}$ & \multicolumn{2}{|c|}{$G C_{i}$} \\
\hline$r r$ & 1.00 & 0.00 \\
$r w$ & 0.50 & 0.50 \\
$w w$ & 0.00 & 1.00 \\
& & \\
\hline
\end{tabular}

\begin{tabular}{|ll|ccc|}
\multicolumn{7}{|c}{ table 6} \\
\hline$G C_{1}$ & $G C_{2}$ & $r r$ & $r w$ & $w w$ \\
\hline$r$ & $r$ & 1.00 & 0.00 & 0.00 \\
$r$ & $w$ & 0.00 & 1.00 & 0.00 \\
$w$ & $r$ & 0.00 & 1.00 & 0.00 \\
$w$ & $w$ & 0.00 & 0.00 & 1.00 \\
\hline
\end{tabular}

Conditional probability tables $4-6$, satisfied by all $P \in \mathbb{P}^{3}$

Now consider a distribution $P^{1}\left(V^{3}\right)$ such that

$P^{1}\left(P T_{1}=\right.$ red $)=P^{1}\left(P T_{2}=\right.$ white $)=1.00 .^{31}$ If $P^{1}\left(V^{3}\right) \in \mathbb{P}^{3}$, then $P^{1}\left(P T_{3}=\right.$ pink $)=1.00$. This corresponds to the data, so it may be assumed that $\mathcal{B}^{1}=\left\langle G^{3}, P^{1}\right\rangle$, with $P^{1} \in \mathbb{P}^{3}$, represents and explains this first cross. In the second cross, the $F_{1}$ hybrids are self-fertilized, so let $P^{2}\left(V^{3}\right)$ be such that $P^{2}\left(P T_{1}=p i n k\right)=P^{2}\left(P T_{2}=p i n k\right)=1.00$. If $P^{2}\left(V^{3}\right) \in \mathbb{P}^{3}$, then $P^{2}\left(P T_{3}=\right.$ red $)=0.25, P^{2}\left(P T_{3}=\right.$ pink $)=$ 0.50 , and $P^{2}\left(P T_{3}=\right.$ white $)=0.25$. Hence, the 1:2:1 ratio can be explained by assuming that this cross is rightly described by $\mathcal{B}^{2}=\left\langle G^{3}, P^{2}\right\rangle$, where $P^{2} \in \mathbb{P}^{3}$.

As we saw in section 9, Morgan cites test crosses to confirm the law of segregation. With respect to incomplete dominance, he considers three such crosses: self-fertilization of the red $F_{2}$ plants, selfing of the white $F_{2}$ plants, and selfing of the pink $F_{2}$ hybrids (Morgan [1928], p. 6). All predictions turned out to be true. In other words, for all three crosses there is a $P \in \mathbb{P}^{3}$ such that the corresponding $\mathcal{B}=\left\langle G^{3}, P\right\rangle$ explains the data. As a result, the adequacy of $\mathbb{P}^{3}$ and of $\mathfrak{B}^{3}$ is confirmed.

Flower colour in four-o'clocks is not the only character that shows incomplete dominance. Like results have been obtained with snapdragons (Antirrhinum), where crosses of red- and white-flowered plants give rise to pink-flowered offspring (Klug et al. [2006], pp. 68-9). 
These relations can be generalized to all monohybrid crosses with incomplete dominance. Let $\mathfrak{B}^{\beta}=\left\langle G^{\beta}, \mathbb{P}^{\beta}\right\rangle$, with $G^{\beta}=\left\langle V^{\beta}, E\right\rangle$, be a generic credal net such that $V^{\beta}$ is value-isomorphic to $V^{3}, G^{\beta}$ is value-isomorphic to $G^{3}$, and $\mathfrak{B}^{\beta}$ is distribution-identical to $\mathfrak{B}^{3}$. Any monohybrid cross with incomplete dominance can be described by means of a causal net that is distribution-identical to some member of $\mathfrak{B}^{\beta} \cdot{ }^{32}$ Complete dominance and incomplete dominance are clearly different, as $\mathfrak{B}^{\alpha}$ and $\mathfrak{B}^{\beta}$ are not value-isomorphic, let alone distribution-identical.

\section{Anomalies}

The history of classical genetics is replete with theory changes to account for apparent anomalies. In the beginning of the 20th century, more and more exceptions to the principle of complete dominance were reported. In Darden's terminology, these should be regarded as model anomalies (as opposed to monster anomalies), since they required 'a change in the set of patterns for normal, well-functioning cases. [...] either the alteration of a typical pattern or the addition of one or more new patterns to the set' (Darden [1991], p. 199). ${ }^{33}$ Mendel's theory was not abandoned, but adapted. ${ }^{34}$

In the structuralist vocabulary: crosses with complete dominance and with incomplete dominance are different kinds of intended applications, giving rise to different kinds of data structures, which are to be dealt with by means of different theory-elements (Balzer and Lorenzano [2000], pp. 256-61).

In my terminology: failures of complete dominance gave rise to data for which there is no $\mathcal{B}=\langle G, P\rangle$ such that (i) $\mathcal{B}$ is distribution-identical to some member of $\mathfrak{B}^{\alpha}$, the then available generic credal net, and such that 
(ii) $P$ is consistent with the data. They were model anomalies since they required a new generic credal net, $\mathfrak{B}^{\beta}=\left\langle G^{\beta}, \mathbb{P}^{\beta}\right\rangle$, that substantially differed from $\mathfrak{B}^{\alpha}$.

\section{Multihybrid Crosses with Independent Assortment}

After presenting two monohybrid crosses with complete dominance, and one with incomplete dominance, Morgan turns to dihybrid crosses. He cites one of Mendel's well-known dihybrid crosses with Pisum plants on albumen colour (yellow versus green) and seed shape (round versus wrinkled). ${ }^{35}$ Mendel had first crossed peas with yellow and round seeds and peas with green and wrinkled seeds. The resulting hybrids $\left(F_{1}\right)$ were yellow and round. Then he self-fertilized the hybrids, thus obtaining $F_{2}$ plants which were yellow round, yellow wrinkled, green round, and green wrinkled in the ratio of 9:3:3:1. (Morgan [1928], pp. 7-8)

To explain the phenotypic distributions in the $F_{1}$ and the $F_{2}$ generation, Morgan takes the law of segregation and adds the assumption that segregation for one pair of elements is independent of segregation for another pair. This is the law of independent assortment (Morgan [1928], p. 10). He assumes complete dominance for the pairs yellow/green and round/wrinkled, as in the monohybrid crosses. He adopts some revised version of the combination principle (see figure 8 in Morgan [1928], p. 9). Finally, he assumes chance fertilization. Together, these laws or explanatory principles give rise to a credal net, say $\mathfrak{B}^{4}=\left\langle G^{4}, \mathbb{P}^{4}\right\rangle$, the members of which explain dihybrid crosses on albumen colour and seed shape in pea plants. Let us see how $\mathfrak{B}^{4}$ looks like.

Clearly, the above crosses cannot be described by any set of variables that is value-isomorphic to $V^{\alpha}$ or $V^{\beta}$. Each phenotypic variable has four 
possible values: $\left[P T_{i}\right]=\{$ yellowround, yellowwrinkled, greenround, greenwrinkled\}. Morgan refers these phenotypes to eight possible genotypes: $\left[G T_{i}\right]=\{G G W W, G G W w, \ldots, g g w w\}$; see table 7 for the full list. The gametes have four possible make-ups: $\left[G C_{i}\right]=\{G W, G w, g W$, $g w\} .{ }^{36}$ ( $G$ stands for the yellow-producing allele, $g$ for green, $W$ for round, $w$ for wrinkled.) Let $V^{4}$ be the set of these variables and let $G^{4}=\left\langle V^{4}, E\right\rangle$ be isomorphic to figure 1 .

Tables 7 and 8 specify some of the constraints to be satisfied by all $P$ in the credal set $\mathbb{P}^{4}$ over $V^{4}$. (I will not discuss the combination principle, the specification of which is straightforward. Nor will I discuss chance fertilization, which is ingrained in figure 1.) Table 7 explicates the principle of complete dominance for both albumen colour and seed shape. Table 8 explicates the law of segregation and the law of independent assortment. The fifth line of table 8 shows the quintessence of independent assortment: $P\left(G C_{i}=G W \mid G T_{i}=G g W w\right)=P\left(G C_{i}=G w \mid G T_{i}=G g W w\right)=$ $P\left(G C_{i}=g W \mid G T_{i}=G g W w\right)=P\left(G C_{i}=g w \mid G T_{i}=G g W w\right)=\frac{1}{4}$ (compare this with the fifth line of table 10).

Both aforecited crosses $(G G W W \times g g w w$ and $G g W w \times G g W w)$ can be explained by means of a causal net $\mathcal{B} \in \mathfrak{B}^{4}$. Similar crosses can be explained in a similar way. Hence, let $\mathfrak{B}^{\gamma}=\left\langle G^{\gamma}, \mathbb{P}^{\gamma}\right\rangle$ be the generic credal net for dihybrid crosses with complete dominance and independent assortment, where $G^{\gamma}=\left\langle V^{\gamma}, E\right\rangle$ and where $\mathfrak{B}^{\gamma}$ is distribution-identical to $\mathfrak{B}^{4}$.

\section{Multihybrid Crosses with Linkage and Crossing-over}

Many cases of independent assortment were known at the time, yet independent assortment is not a universally applicable law. 
table 7

\begin{tabular}{|l|cccc|}
\hline & \multicolumn{4}{|c|}{$P T_{i}$} \\
$G T_{i}$ & $y e$ & $y e$ & $g r$ & $g r$ \\
$G G W W$ & 1.00 & 0.00 & 0.00 & 0.00 \\
$G G W w$ & 1.00 & 0.00 & 0.00 & 0.00 \\
$G G w w$ & 0.00 & 1.00 & 0.00 & 0.00 \\
$G g W W$ & 1.00 & 0.00 & 0.00 & 0.00 \\
$G g W w$ & 1.00 & 0.00 & 0.00 & 0.00 \\
$G g w w$ & 0.00 & 1.00 & 0.00 & 0.00 \\
$g g W W$ & 0.00 & 0.00 & 1.00 & 0.00 \\
$g g W w$ & 0.00 & 0.00 & 1.00 & 0.00 \\
$g g w w$ & 0.00 & 0.00 & 0.00 & 1.00 \\
\hline
\end{tabular}

table 8

\begin{tabular}{|l|cccc|}
\hline \multicolumn{5}{|c|}{ table 8 } \\
\hline & \multicolumn{5}{|c|}{$G C_{i}$} \\
$G T_{i}$ & $G W$ & $G w$ & $g W$ & $g w$ \\
\hline$G G W W$ & 1.00 & 0.00 & 0.00 & 0.00 \\
$G G W w$ & 0.50 & 0.50 & 0.00 & 0.00 \\
$G G w w$ & 0.00 & 1.00 & 0.00 & 0.00 \\
$G g W W$ & 0.50 & 0.00 & 0.50 & 0.00 \\
$G g W w$ & 0.25 & 0.25 & 0.25 & 0.25 \\
$G g w w$ & 0.00 & 0.50 & 0.00 & 0.50 \\
$g g W W$ & 0.00 & 0.00 & 1.00 & 0.00 \\
$g g W w$ & 0.00 & 0.00 & 0.50 & 0.50 \\
$g g w w$ & 0.00 & 0.00 & 0.00 & 1.00 \\
\hline
\end{tabular}

Conditional probability tables $7-8$, to be satisfied by all $P \in \mathbb{P}^{4}$ (together with the combination principle)

It might, then, have seemed justifiable to extend this

conclusion to as many pairs of characters as enter any

particular cross. This would mean that there are as many

independent pairs of elements in the germinal material as there

are possible characters. Subsequent work has shown, however,

that Mendel's second law of independent assortment has a

more restricted application, since many pairs of elements do

not assort freely, but certain elements that enter together show

a tendency to remain together in succeeding generations. This

is called linkage. (Morgan [1928], p. 10)

More precisely,

By linkage we mean that when certain characters enter a cross together, they tend to remain together in later generations, or, stated in a negative way, certain pairs of characters do not assort at random. (Morgan [1928], p. 10)

Genes that are linked belong to the same linkage group. Drosophila melanogaster (fruit fly), for example, has four linkage groups (Morgan 
[1928], pp. 11-2). It was soon discovered that genes in the same linkage group are not always completely linked. There may be some interchange between linkage groups.

This interchange is called crossing-over, which means that, between two corresponding linked series, there may take place an orderly interchange involving great numbers of genes.

(Morgan [1928], p. 14)

As an exemplar, Morgan cites crosses performed by Bateson and Punnett on Lathyrus odoratus or sweet peas (purple flowers and long pollen grains crossed to red flowers and round pollen grains). Flower colour and pollen shape in sweet peas were known to show normal Mendelian segregation and to satisfy the principle of complete dominance, with purple dominant to red and long dominant to round (Darden [1991], pp. 122-3). Bateson and Punnett had observed that 'the two types that go in together come out together more frequently than expected for independent assortment of purple-red and round-long' (Morgan [1928], p. 10).

Because of the difference between linkage and independent assortment, there is no credal net $\mathfrak{B}$ which is (i) distribution-identical to $\mathfrak{B}^{\gamma}$, the generic credal net for dihybrid crosses with independent assortment, and which is such that (ii) its members can be used to explain dihybrid crosses on flower colour and pollen shape in sweet peas. We should seek a new credal net, say $\mathfrak{B}^{5}=\left\langle G^{5}, \mathbb{P}^{5}\right\rangle$, with $G^{5}=\left\langle V^{5}, E\right\rangle$ and such that $\mathbb{P}^{5}$ accounts for the failure of independent assortment.

Let us first have a look at Bateson and Punnett's data (Morgan [1928], p. 11, figure 9). ${ }^{37}$ Cross 1: Sweet peas with purple flowers and long pollen grains were crossed with sweet peas with red flowers and round 
pollen grains. (It may be assumed that all plants were true-breeding.) The resulting hybrids $\left(F_{1}\right)$ had long pollen grains and purple flowers. Cross 2: Self-fertilization of the $F_{1}$ generation produced $F_{2}$ individuals in the following proportions (the absolute frequencies are Morgan's, I have added the percentages):

cross 2: long, purple round, purple long, red round, red

$\begin{array}{rrrr}583 & 26 & 24 & 170 \\ 73 \% & 3 \% & 3 \% & 21 \%\end{array}$

These results can be explained as follows. We know that the $F_{1}$ plants are hybrid, so $P\left(G T_{1}=G g W w\right)=P\left(G T_{2}=G g W w\right)=1.00$, where $G$ denotes the purple-producing allele, $g$ the red-producing allele, $W$ the long-producing allele and $w$ the round-producing allele. ${ }^{38}$ We may assume that complete dominance still holds between purple and red, and between long and round in the dihybrid case, so that we may rely on some variant of table 7. We may also assume that the combination principle holds.

Then all we need to do is to find the appropriate conditional probability table for the relation between $G T_{i}$ and $G C_{i}$. It turns out that the phenotypic distribution for the $F_{2}$ individuals can be explained if (but not only if, see below)

$$
\begin{aligned}
P\left(G C_{i}=G W \mid G T_{i}=G g W w\right) & =0.46 \\
P\left(G C_{i}=G w \mid G T_{i}=G g W w\right) & =0.04 \\
P\left(G C_{i}=g W \mid G T_{i}=G g W w\right) & =0.04 \\
P\left(G C_{i}=g w \mid G T_{i}=G g W w\right) & =0.46
\end{aligned}
$$

For example, by the principle of complete dominance the $F_{2}$ individuals can only have round pollen and red flowers if their genotype is ggww. Hence their fertilizing gametes must both be $g w$. The following 
calculations retrodict that $21 \%$ of the $F_{2}$ plants will have round pollen and red flowers (assuming chance fertilization). We know that $P\left(G T_{1}=G g W w\right)=P\left(G T_{2}=G g W w\right)=1.00$. By the conditional probabilities just given, we can compute that $P\left(G C_{1}=g w\right)=0.46$, and likewise that $P\left(G C_{2}=g w\right)=0.46$. Given chance fertilization, $P\left(G C_{1}=g w \wedge G C_{2}=g w\right)=0.46 \times 0.46=0.21$. So by the combination principle and by some analogue of table $7, P\left(G T_{3}=g g w w\right)=0.21$ and $P\left(P T_{3}=\right.$ roundred $)=0.21$. Like calculations allow to retrodict the probabilities of the other phenotypes in $F_{2} \cdot{ }^{39}$

$\mathbb{P}^{5}$ cannot be specified by demanding that for all $P \in \mathbb{P}^{5}$ the above conditional probabilities hold, since these conditional probabilities are cross-dependent. Assume that, in cross 1, GGww individuals had been crossed with $g g W W$ individuals (instead of $G G W W \times g g w w) .{ }^{40}$ The resulting $F_{1}$ hybrids would all be $G g W w$, as in Morgan's example. But self-fertilization of these hybrids would give $F_{2}$ plants in the following proportions:

long, purple round, purple long, red round, red

$$
50,16 \% \quad 24,84 \% \quad 24.84 \% \quad 0.16 \%
$$

The reason is that here $G$ and $w$ (and thus $g$ and $W$ ), tend to remain together, whereas in the original cross $G$ and $W$ (and thus $g$ and $w$ ) did so:

$$
\begin{aligned}
P\left(G C_{i}=G W \mid G T_{i}=G g W w\right) & =0.04 \\
P\left(G C_{i}=G w \mid G T_{i}=G g W w\right) & =0.46 \\
P\left(G C_{i}=g W \mid G T_{i}=G g W w\right) & =0.46 \\
P\left(G C_{i}=g w \mid G T_{i}=G g W w\right) & =0.04
\end{aligned}
$$

How, then, should we characterize $\mathbb{P}^{5}$ ? Linkage and crossing-over influence the probabilistic relations between $G T_{i}$ and $G C_{i}$, as compared to 
table 9

\begin{tabular}{|l|cccc|}
\hline & \multicolumn{4}{|c|}{$P T_{i}$} \\
$G T_{i}$ & lng & rnd & lng & rnd \\
\hline$G G W W$ & 1.00 & 0.00 & 0.00 & 0.00 \\
$G G W w$ & 1.00 & 0.00 & 0.00 & 0.00 \\
$G G w w$ & 0.00 & 1.00 & 0.00 & 0.00 \\
$G g W W$ & 1.00 & 0.00 & 0.00 & 0.00 \\
$G g W w$ & 1.00 & 0.00 & 0.00 & 0.00 \\
& & & & \\
$G g w w$ & 0.00 & 1.00 & 0.00 & 0.00 \\
$g g W W$ & 0.00 & 0.00 & 1.00 & 0.00 \\
$g g W w$ & 0.00 & 0.00 & 1.00 & 0.00 \\
$g g w w$ & 0.00 & 0.00 & 0.00 & 1.00 \\
\hline
\end{tabular}

table 10

\begin{tabular}{|l|llll|}
\hline & \multicolumn{5}{|c|}{$G C_{i}$} \\
$G T_{i}$ & $G W$ & $G w$ & $g W$ & $g w$ \\
\hline$G G W W$ & 1.00 & 0.00 & 0.00 & 0.00 \\
$G G W w$ & 0.50 & 0.50 & 0.00 & 0.00 \\
$G G w w$ & 0.00 & 1.00 & 0.00 & 0.00 \\
$G g W W$ & 0.50 & 0.00 & 0.50 & 0.00 \\
$G g W w$ & $\frac{a}{2}$ & $\frac{1-a}{2}$ & $\frac{1-a}{2}$ & $\frac{a}{2}$ \\
& $a \in\{0.08,0.92\}$ & \\
$G g w w$ & 0.00 & 0.50 & 0.00 & 0.50 \\
$g g W W$ & 0.00 & 0.00 & 1.00 & 0.00 \\
$g g W w$ & 0.00 & 0.00 & 0.50 & 0.50 \\
$g g w w$ & 0.00 & 0.00 & 0.00 & 1.00 \\
\hline
\end{tabular}

Conditional probability tables $9-10$, satisfied by all $P \in \mathbb{P}^{5}$ (together with the combination principle). Table 9 is analogous to table 7 (complete dominance holds, even though pollen shape is linked with flower colour). The difference between tables 8 and 10 reveals the difference between independent assortment on the one hand, and linkage and crossing-over on the other hand. $\min \{0.08,0.92\}$ is the frequency of crossing-over.

cases of independent assortment, if and only if $G T_{i}=G g W w$ (i.e., if and only if doubly heterozygous plants are involved). ${ }^{41}$ Both sets of conditional probabilities listed above can be summarized as follows:

$$
\begin{aligned}
P\left(G C_{i}=G W \mid G T_{i}=G g W w\right) & =\frac{a}{2} \\
P\left(G C_{i}=G w \mid G T_{i}=G g W w\right) & =\frac{1-a}{2} \\
P\left(G C_{i}=g W \mid G T_{i}=G g W w\right) & =\frac{1-a}{2} \\
P\left(G C_{i}=g w \mid G T_{i}=G g W w\right) & =\frac{a}{2},
\end{aligned}
$$

where $a \in\{0.08,0.92\}$ and where $\min \{0.08,0.92\}=0.08$ is the frequency of crossing-over for dihybrid crosses on flower colour and pollen shape in Lathyrus odoratus. All this is summarized in table 10.

$\mathbb{P}^{5}$ thus is the set of distributions over $V^{5}$ that satisfy tables 9 and 10 (plus the combination principle and chance fertilization). (Note that the physical probability a may take two possible values and that hence the distribution over $G C_{i}$ conditional on $G T_{i}$ is imprecise. But regarding 
crosses on flower colour and pollen shape in Lathyrus odoratus, the physical probability a may not take a value between 0.08 and 0.92 .)

Other crosses, for example on Drosophila, also revealed linkage and crossing-over, but with different frequencies of crossing-over. The frequency of crossing-over for some particular pair of genes in some particular organism is most easily determined observationally as follows (cf. Morgan [1928], pp. 14-7). Let $A$ and $B$ denote dominant alleles; $a$ and $b$ their recessive counterparts. Let two grandparental individuals (e.g. $A A B B$ and $a a b b$, or $A A b b$ and $a a B B$ ) together produce a double heterozygote $(A a B b)\left(F_{1}\right)$. Perform the cross $A a B b \times a a b b$ (the resulting offspring is $F_{2}$ ). Barring problems of statistical inference and barring cases of double crossing-over, the frequency of crossing-over is identical to the proportion of individuals in $F_{2}$ that do not phenotypically resemble any of the grandparents (called cross-over types).

Crosses on wing colour (yellow, gray) and eye colour (white, red) in Drosophila revealed a frequency of crossing-over of $1 \%$. Other crosses in Drosophila gave other frequencies: $33 \%$ in white versus red eyes and miniature versus long wings, or $40 \%$ in white versus red eyes and forked versus normal bristles. If there are no cross-over types, linkage is complete.

In short, Morgan and his group observed all possible percentages of crossing-over, up to nearly 50\% (Morgan [1928], pp. 19-20). As a result, the generic credal net for dihybrid crosses with linkage and crossing-over (and with complete dominance) should look like the following. Let $\mathfrak{B}^{\delta}=\left\langle G^{\delta}, \mathbb{P}^{\delta}\right\rangle$ be such that (i) $G^{\delta}=\left\langle V^{\delta}, E\right\rangle$ is value-isomorphic to $G^{5}=\left\langle V^{5}, E\right\rangle$, and (ii) any $P \in \mathbb{P}^{\delta}$ satisfies complete dominance, the combination principle and some analogue of table 10 , where $a \in\{b, 1-b\}$ for some $b \in[0.00,0.50]$. (It should be noted that the characterization of 
$\mathbb{P}^{\delta}$ should invoke one more principle relating to the linear ordering of the genes, see section 16.)

\section{Double Crossing-over and the Linear Order of the Gene}

Crossing-over may occur within linkage groups and the frequency of crossing-over may be estimated from the types of crosses cited in section 15. However, the Morgan group discovered a phenomenon called double crossing-over that leads to a systematic underrating of the frequencies of crossing-over. 'By double crossing-over is meant that interchange takes place twice between two pairs of genes involved in the cross. The result is to lower the observed cases of crossing-over, since a second crossing-over undoes the effect of a single crossing-over' (Morgan [1928], p. 20, original emphasis). This problem is solved by taking into account more than two pairs of traits.

For example, if a female [Drosophila] with the following nine characters of Group I, scute, echinus, cross-veinless, cut, tan, vermilion, garnet, forked and bobbed, is crossed to a wild type male, and if the $F_{1}$ female [...] is back-crossed to the same multiple recessive type, the offspring produced will give a record of every crossing-over. (Morgan [1928], p. 20)

Based on these results, Morgan draws a linear diagram depicting the corresponding genes in the linkage group ([1928], p. 21). In figure 2, crossing-over has taken place twice. As a result, it seems as if no crossing-over has taken place between e.g. forked and cross-veinless, thus lowering the estimated frequency of crossing-over. By taking the intermediate loci into account, this bias is remedied. 

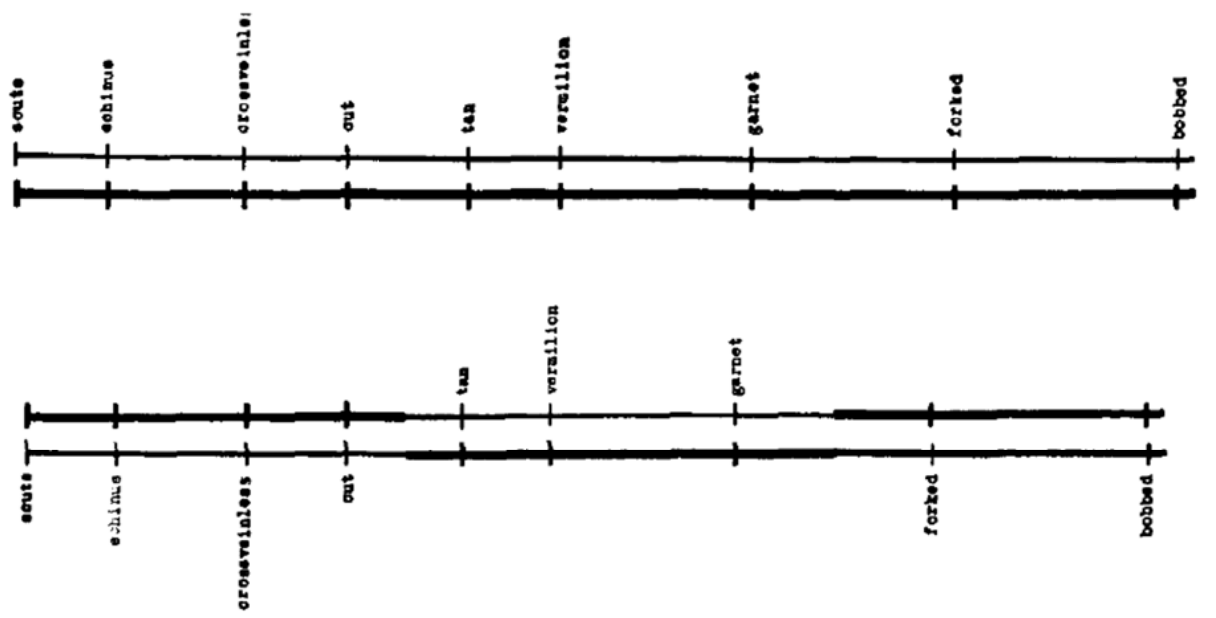

Figure 2: The linear order of the genes in Group I of Drosophila (adapted from Morgan [1928], p. 21, figures 15 and 18). At the top, no crossing-over has occurred. At the bottom, crossing-over took place twice (between tan and cut, and between garnet and forked).

More generally, the Morgan group concluded that linkage groups can be represented linearly, with genes lying in a line 'like beads on a string' (Morgan [1928], p. 24). Evidence for this linear order came from two sources. First, cytological evidence showed that chromosomes were threadlike entities (Morgan [1928], pp. 38-44). As the assumption that genes are located on the chromosomes became more and more accepted, it was most natural to assume they are organized linearly. Second, genetic evidence pointed in the same direction in a way that is relevant for the characterization of $\mathbb{P}^{\delta}$.

It can be assumed that crossing-over is the result of some interchange taking place at the level of the chromosomes (Morgan [1928], p. 39). Though cytological evidence for such an interchange was not conclusive, it was quite convincing (Morgan [1928], p. 44). Such considerations gave rise to the concept of map distance: the 'distance' between pairs of elements or 
genes on the same chromosome, measured in terms of their percentage of crossing-over (Morgan [1928], p. 22). ${ }^{42}$ The concept of map distance allowed to further corroborate the hypothesis of the linear order of the genes.

Suppose that crossing-over between yellow wings and white eyes occurs in 1.2 per cent of cases. If we then test white with a third member of the same series, such as bifid wings, we find 3.5 per cent of crossing-over [...]. If bifid is in line and on one side of white it is expected to give with yellow 4.7 per cent crossing-over, if on the other side of white it is expected to give 2.3 per cent of crossing-over with yellow. In fact, it gives one of these values, namely, 4.7. We place it, therefore, below white in the diagram. This sort of result is obtained whenever a new character is compared with two other members of the same linkage group. The crossing-over of a new character is found to give, in relation to two other known factors, either the sum or the difference of their respective cross-over values. This is the known relation of points on a line, and is the proof of the linear order of the genes; for no other spatial relation has yet been found that fulfills these conditions. (Morgan [1928], p. 24, my emphasis)

Morgan's citation can be explicated as follows. Let $i, j$ and $k$ be characters of the same kind of organism, say Drosophila. Let the credal net $\mathfrak{B}^{i j}=\left\langle\left\langle V^{i j}, E\right\rangle, \mathbb{P}^{i j}\right\rangle$ be the set of causal nets that represent dihybrid crosses on $i$ and $j$ (and analogously for $\mathfrak{B}^{j k}=\left\langle\left\langle V^{j k}, E\right\rangle, \mathbb{P}^{j k}\right\rangle$ and $\left.\mathfrak{B}^{i k}=\left\langle\left\langle V^{i k}, E\right\rangle, \mathbb{P}^{i k}\right\rangle\right)$. If $i, j$ and $k$ are cases of complete dominance, then $\mathfrak{B}^{i j}, \mathfrak{B}^{j k}$ and $\mathfrak{B}^{i k}$ are distribution-identical to $\mathfrak{B}^{\delta}{ }^{43}$ Finally, let 
$a^{i j}, a^{j k}$, and $a^{i k}$ be the frequencies of crossing-over for $\mathfrak{B}^{i j}, \mathfrak{B}^{j k}$ and $\mathfrak{B}^{i k}$.

If the genes belong to the same linkage group, then according to Morgan's findings either $a^{i j}=a^{j k}+a^{i k}$ or $a^{i j}=\left|a^{j k}-a^{i k}\right|$. This imposes an important constraint (in the structuralists' sense) on the characterization of $\mathbb{P}^{\delta}$ : it expresses important connections between different local applications of $\mathfrak{B}^{\delta}$.

\section{Causal-Structural Explanation}

Let us turn now to the final part of this paper: the causal-structural account of explanation and its relation to unification. As we saw in sections 9-16, Morgan explains filial phenotypic distributions by means of a carefully chosen set of explanatory principles or laws and assumptions regarding the parental genotypes. As such, his explanations are a syntactic enterprise, a matter of derivation. But they can also be explicated model-theoretically. To explain a given phenotypic distribution, one tries to find an appropriate credal net $\mathfrak{B}$, satisfying the right explanatory principles, so that one of its causal nets can be used to embed the data in. The label 'causal-structural account of explanation' covers both these aspects of explanation.

This account has a number of interesting characteristics. First and foremost, it is a causal account of explanation. The laws or explanatory principles invoked by Morgan are inextricably joined to the causal structure in figure 1, where this structure is defined in terms of Woodward's (DC).

It may seem strange to equate the explanatory principles of classical genetics with laws. After all, aren't laws of nature true, universal and physically necessary generalizations whereas the explanatory principles of 
classical genetics face numerous exceptions, have a limited domain of application and are highly contingent? No. By endorsing this claim, one would demand too much from laws of nature - in fact so much that it becomes questionable whether there are any laws at all in the special sciences. As I have argued following Mitchell ([1997], [2000]), explanatory principles such as those of classical genetics, need not satisfy the traditional criteria for lawfulness to deserve the label 'law' (Leuridan [2010]). If they (or, more precisely, the regularities they describe) have sufficient stability and strength, ${ }^{44}$ and if they are invariant under some range of interventions, they can be used (in particular contexts) for explanation (even if they fail to hold outside those contexts). The causal-structural account of scientific theories provides a nice explication of this view. The explanatory principles or laws of each (generic) credal net have a limited domain of application. Within that domain, they may be used for explanation regardless of the fact that they face exceptions (anomalies, see section 13) and fail to hold in other credal nets.

Another way to phrase this is as follows. Each generic credal net implicitly incorporates a set of ceteris paribus conditions which help to determine its domain of intended applications. Of course, these are not just any ceteris paribus conditions, but well delineated sets of such conditions, based on existing scientific knowledge. So it should not be feared that these ceteris paribus conditions make the principles of classical genetics trivially true. (See Pietroski and Rey [1995]; Earman et al. [2002]; Woodward [2002]; and Mitchell [2002] for a discussion of ceteris paribus clauses.) For example, $\mathfrak{B}^{\alpha}-\mathfrak{B}^{\delta}$ all assume that mutation does not occur; and $\mathfrak{B}^{\gamma}$ and $\mathfrak{B}^{\delta}$ both assume that there is no gene interaction.

A second characteristic of the causal-structural account of explanation 
is that it also is an epistemic and representational, rather than an ontic account of explanation. What explains is not the causal structure of the world itself, but causal explanatory principles describing that causal structure. The model-theoretic approach need not detract from that representational character, as classical genetics' causal nets are themselves 'simulacra' of real-world states of affairs (cf. Cartwright [1983], pp.

143-62). ${ }^{45}$ To say it with a catchword: 'No explanation without representation!' Yet focussing on these models also helps to see that explanation is not merely derivational; the causal nets in question have to bear the right relations to the ontic structure of the world. And it helps to illuminate the notion of explanatory unification; the causal nets in question bear interesting similarity relations to each other. Finally, the formal framework I use allows for an integrated approach to both the qualitative and the quantitative aspects of causal structures in a way that is suitable for e.g. prediction (see section 11).

\section{Explanatory Unification}

The causal-structural account of scientific theories allows us to get a grip on explanatory unification within a causalist framework. Today, Kitcher's anti-causalist approach is still influential in the literature on unification. Yet in the past years, the need for, or desirability of, a causalist account has been stressed by several authors. Here, I will present the criticisms raised by Mäki ([2001]) and Woodward ([2003]) against Kitcher's unificationism, elaborate my own proposal, and contrast it with Strevens' kairetic account.

Kitcherian unification consists in showing that many different phenomena can be derived from a small number of explanatory patterns. 
The unifying power of a set of argument patterns varies directly with the number of statements that can be derived by means of its members, directly with the stringency of the patterns in the set, ${ }^{46}$ and inversely with the number of patterns in the set (Kitcher [1989], p. 435). The patterns in the most unifying set of patterns over a body of scientific knowledge $K$ (called the explanatory store $E(K)$ ) determine what explains what.

Both Mäki and Woodward endorse the importance of unification as an epistemic virtue, but they are dissatisfied by Kitcher's approach. I go with them in several ways. I, too, consider unificatory power an epistemic virtue. It is a virtue which scientific theories may have to a greater or lesser extent on top of their being explanatory. I also agree with their critique of Kitcher. Yet I am not fully satisfied with their views either (for reasons to be outlined below). As I will show, the causal-structural theory of explanation adequately addresses their worries while providing an interesting and elaborate alternative to Kitcher's account.

Woodward finds the idea that explanation is a matter of unifying a range of different phenomena 'unquestionably intuitively appealing'; generality is 'at least sometimes an explanatory virtue'; and 'theory unification has clearly played an important role in science' ([2003], p. 358). Yet he wonders whether our intuitive notion(s) of unification can be made more precise in a way that fits our interventionist intuitions concerning explanation ([2003], p. 358). In his opinion, Kitcher fails to do so. Unification may be an explanatory virtue, but it is not a sufficient condition for explanation: 'considerations having to do with unification do not automatically pick out those derivations that are explanatory from those that are not' ([2003], p. 361).

Woodward's own proposal is to explicate unification in terms of ranges 
of invariance. A generalization's explanatory depth is tied to the range of ideal interventions under which it is invariant: the wider its range of invariance, the more explanatory it is ([2003], pp. 257-65). This range of invariance is also related to the generalization's generality or unifying power ([2003], pp. 366 and 373).

I agree that 'range of invariance' captures part of the notion of unification, but I think that more of Kitcher's intuitions can be recovered in the interventionist approach (note that Woodward leaves open this possibility; [2003], p. 373). More specifically, generic credal nets and their possible interrelations allow us to explicate the intuition that different phenomena can be explained by means of a number of more or less similar causal 'patterns'. But let me first turn to Mäki's views.

For Mäki, Kitcher's account of unification is problematic as it comes down to mere derivational unification, i.e. 'unification as a derivational accomplishment without ontological groundings' ([2001], p. 497). According to Kitcher, explanation is not a matter of describing causal relations in the world. Instead, causal relevance is dependent on explanatory relevance and hence on derivational unification ([1989], pp. 436 and 499; see also section 4 above.

In Mäki's opinion, unification should not be (merely) derivational; it should (also) be ontological. ${ }^{47}$ Ontological unification is 'based on the referential and representational capabilities of theories', not on their inferential capabilities (Mäki [2001], p. 498). ${ }^{48}$ It is 'a matter of redescribing apparently independent and diverse phenomena as manifestations $[\ldots]$ of one and the same small number of entities, powers, and processes' (Mäki [2001], p. 498).

Unification, according to this picture, is not just a matter of 
derivational success but rather a matter of successfully

representing how things are related in the causal order of things

in the world. (Mäki [2001], p. 500, my emphasis)

What is important for the present paper, is the fact that derivational and ontological unification need not be incompatible (Mäki [2001], p. 499). They may coincide, or derivational unification may have partial ontological grounds. Ontological unification is only incompatible with mere derivational unification. I agree that ontological and derivational unification may coincide. But Mäki's view is only partly satisfying, as he adds the following:

My hunch is that this is a contingent issue; there is no necessity for the two kinds of unification to be related in one particular way or another. (Mäki [2001], p. 499)

Mäki's hunch is plausible, provided derivation is tied to standard logical inference. In this respect I would like to take his side. Kitcher's argument patterns are phrased in natural language, but their expressive power does not exceed that of non-modal second order logic joined with probability theory. ${ }^{49}$ Hence, whether they coincide with 'the causal order of things in the world' (where this causal order is not defined as a function of the explanatory relations), is a contingent issue. Yet if derivation is tied to causal reasoning, the tie between derivational and ontological unification is much stronger and Mäki's distinction largely dissolves. In this sense, his answer is but partly satisfying.

In my causal-structural framework, unification is a matter of similarity between a theory's intended applications and, relatedly, between its causal and credal nets. As such, it comes in degrees. The stronger the 
relations between credal nets (where distribution-identity is stronger than value-isomorphism, which is stronger than isomorphism), the stronger the corresponding phenomena are unified. But, and this is very important, all credal nets are isomorphic.

First, different crosses on a particular trait (or set of traits) in a particular organism are explained by means of a given set of explanatory principles. For example, crosses on stem length in pea plants are explained by means of complete dominance, the law of segregation, chance fertilization, and the combination principle. Semantically speaking, these different crosses are embedded in different causal nets $\mathcal{B}^{1}, \mathcal{B}^{2}, \ldots$ which belong to a common credal net $\mathfrak{B}^{1}$. This already provides some unification.

Second, other crosses, e.g. on eye colour in humans, are explained by means of the very same explanatory principles. They are embedded in causal nets that belong to a different credal net, such as $\mathfrak{B}^{2} . \mathfrak{B}^{2}$ is distribution-identical to $\mathfrak{B}^{1}$, which means that inheritance of stem length in pea plants and of eye colour in humans is highly similar, notwithstanding the fact that prima facie they are very distinct characters in very distinct species. This can be generalized to all monohybrid crosses with complete dominance. These can be explained by members of credal nets that are distribution-identical to $\mathfrak{B}^{1}$. I have used the generic credal net $\mathfrak{B}^{\alpha}$ as an abstract representation of all such crosses. Many divergent cases can be viewed as highly similar (in the sense of distribution-identity). Distribution-identity is a very strong notion of similarity which expresses a kind of very strong unification. It shows that phenomena not only resemble each other qualitatively (qua qualitative causal relations), but also quantitatively or probabilistically.

Third, monohybrid crosses with complete dominance are but one kind 
or type of phenomena. Other kinds were explained by means of other sets of explanatory principles, giving rise to other generic credal nets. (Each of these generic credal nets, and their instances, provide unification for their domain of intended application.) I have discussed $\mathfrak{B}^{\beta}, \mathfrak{B}^{\gamma}, \mathfrak{B}^{\delta}$, and more could be specified for other types of crosses (see section 19). All these credal nets (and their specific instances) are isomorphic, as their graphs are isomorphic to figure 1. Isomorphism is a much weaker relation than distribution-identity, and hence expresses a weaker notion of unification, but the large number of kinds of phenomena that stand in this isomorphism relation made classical genetics interestingly unifying. And what is important: it shows that all these kinds of crosses are explained in terms of a common underlying causal structure, and that the distinct generic credal nets of classical genetics each characterize very similar mechanisms $^{50}$ (Darden [1991], p. 19) or very similar entities, powers and processes (Mäki [2001], p. 498).

The causal-structural notion of unification is both derivational and ontological and hence meets Mäki's worries. It is derivational because classical genetics makes use of (a limited stock of) explanatory principles, which are syntactic statements, to explain a wide range of phenomena. It is also ontological, because these principles are closely tied to a common causal mechanism (entities, processes, powers) which is qualitatively represented in figure $1 .{ }^{51}$

All of classical genetics' causal nets are isomorphic, and this shows that apparently very different phenomena can be explained in a unified way. Yet I do not claim that isomorphism of a theory's causal nets is a sufficient condition for that theory to be unifying. One may, after all, devise artifacts that have a causal structure that is isomorphic to figure 1 
but which have nothing to do with classical genetics. To show that their structure is isomorphic to the causal nets of classical genetics would only be a kind of 'spurious unification' (Kitcher [1981], pp. 526-9). The phenomena to be explained have to be in the domain of intended applications of classical genetics. This is not a defect of my account, but an unavoidable feature that it shares with the structuralists and with Kitcher. From their works it is clear that there is no straightforward recipe, or set of necessary and sufficient conditions, for establishing a theory's domain of intended applications. As I wrote in section 3.1, the structuralist notion of intended applications cannot be defined in a purely formal way; it also relies on pragmatic considerations - see (Balzer et al. [1987], pp. 37-40, 87-9; Balzer and Lorenzano [2000], p. 245; Balzer and Dawe [1986a], p. 67). Sets of intended applications I are often specified by citing a few 'paradigms' or 'exemplars' such as stem length in pea plants. All exemplars of classical genetics concern crosses of organisms with certain phenotypic traits, the resulting progeny of which shows a more or less definite phenotypic distribution. To be included in the set of intended applications of classical genetics, crosses of organisms have to be sufficiently similar to one of its exemplars (qua types of traits involved, qua resulting distributions, etc.). ${ }^{52}$ What counts as sufficiently similar depends on pragmatic considerations. Moreover, the set of classical genetics' intended applications changed over time and frequently was a matter of debate (Darden [1991], pp. 166 and 260). Likewise, Kitcher's notion of filling instructions, which he needs to avoid spurious unification, cannot be formally defined. Although he nowhere says so, it is clear from his writings that their specification has to rely on pragmatic considerations as well.

Isomorphism is not a necessary condition either. Define isomorphism 
class as a class of causal nets that are isomorphic to each other. While classical genetics has only one isomorphism class (all of its causal nets are isomorphic to each other), there may be scientific theories that have more than one such class and still are judged to have at least some unifying power. Yet I conjecture that, ceteris paribus, the less isomorphism classes a theory has, the more we would judge it to be unifying. A theory with less isomorphism classes posits less fundamentally different causal structures underlying the phenomena to be explained. ${ }^{53}$ Thus, isomorphism for causal and credal nets and its stronger nephew, distribution-identity, serve to explicate our intuitive notion of unification in a way that fits the interventionist theory of causation and allows to reconcile derivational with ontological unification.

Let me conclude this section by briefly turning to Strevens' kairetic account of explanation. The claim that the causal-structural account of scientific theories allows us to get a grip on explanatory unification within a causalist framework may suggest that this account is similar in spirit to Strevens' ([2004]), at least prima facie. After all, Strevens also endeavours to 'unify the causal and unificatory approaches to explanation-causally' (see the title of his paper). More specifically, his goal is 'a causal account of explanation that has many of the advantages of the unification account' (Strevens [2004], p. 154). Yet there are some important differences which reward further examination. (Strevens [2004] focuses on explanations of events or singular facts, whereas I have been focusing here on explanations of regularities, yet the differences I mention carry over to the kairetic account of the explanation of laws and regularities; see Strevens [2008], part III.)

A first difference is that Strevens' use of the unificationist framework 
is not meant to capture the notion of unification, but the distinct notion of explanatory relevance. A second, minor difference is that Strevens' use of 'model' as a 'set of propositions' is a syntactic one ([2004], p. 163), whereas mine is semantic. This brings me to a last and most important difference. Strevens does not attempt to explicate his concept of causation. This places him in a comfortable position - too comfortable, perhaps. It allows him, for instance, to stipulate that logical entailment in causal models represents relations of causal production ([2004], p. 163). The literature on interventionist accounts of causation has shown, however, that one should not skate on thin ice when trying to make logical entailment fit causal relations; see e.g. (Hausman [1998], chapter 8) for an elaborate attempt to model explanations as causal arguments. Strevens ([2004], p. 163) acknowledges that not all entailments represent causal processes. Yet he does not provide even the slightest account of what would make an entailment represent a causal process. The best one finds, is whether or not physical theory (the true theory of everything, whatever that may be) attributes to the premises the power to bring about the conclusion ([2004], p. 165). By opting for a specific and substantive account of causation (Woodward's), I have placed myself in a more difficult and vulnerable situation, since I expose myself to possible counterexamples (see section 19 on the tenability of the Causal Markov Condition).

\section{Concluding Remarks}

In this final section, I would like to make some concluding remarks on three issues. First, I will briefly discuss the parts of classical genetics which I have not explicitly dealt with in this paper. Second, I will consider the applicability of the causal-structural account to theories other than 
classical genetics. What characteristics should a theory have in order to fit the causal-structural account? This brings me to a third and very important issue: the tenability of the Causal Markov Condition.

In this paper, I have discussed some of the best known parts of classical genetics, starting from Morgan's exposition from 1928. At the time, however, many phenomena were known that do not fit any of the generic credal nets I have sketched: cases of sex-linked inheritance, multiple alleles, multiple genes, gene interaction, pleiotropy, lethal alleles, non-diploidy, incomplete penetrance, ... I contend that causal nets, credal nets and generic credal nets can be defined for all these phenomena, based on the graph in figure 1. Hence they fit the causal-structural account of classical genetics.

Apart from classical genetics, which other theories would be suitable for the causal-structural account of scientific theories? In my opinion, the following three criteria are relevant (this list is not intended to be exhaustive). First, the theory in question should be causal in the interventionist sense. The concepts of intervention, invariance and the like should be applicable. Given that Woodward ([2003]) uses examples from a wide range of scientific disciplines, ranging from physics and chemistry, over biology and the biomedical sciences, to economics and social theory, this criterion is not very restrictive. Still, not all scientific theories may be suitable. For example, it has been suggested that the notion of 'ideal intervention' is not applicable to EPR phenomena in quantum mechanics (Hausman and Woodward [1999], pp. 560-70). If that is correct, then my causal-structural account is not suited for quantum mechanics. (See Suárez and San Pedro [2011], pp. 183-7, for a critical evaluation of Hausman and Woodward's claim, however.) 
Second, given the relations between the causal-structural account and Sneedian structuralism, I surmise that a good place to look for further candidate theories would be among the wide range of examples that have been discussed by the structuralists (see Balzer et al. [2000], for a collection of paradigmatic examples). Insofar as these theories fit the interventionist framework, approaching them from a causal-structural rather than structuralist perspective would be advantageous. For example, it would allow us to examine to what extent they give rise to causalist explanatory unification.

A theory that seems to meet both criteria is general equilibrium theory in economics, which pictures the economy as 'a collection of economic agents who make supply and demand decisions over commodities, labour types and assets, in order to further their own interests' and 'studies the equilibrium properties of the economy, so conceived' (Bryant [2010], p. 1). Hamminga and Balzer ([2000]) have analysed this theory within the structuralist framework and Woodward ([2003], pp. 233 and 355-6) suggests that the relations between e.g. supply, demand and prices can be analysed in interventionist terms.

The third criterion is related to the first: the theory should not violate the Causal Markov Condition (CMC), as that condition is one of the most central assumptions of my account. The aforementioned claim by Hausman Woodward ([1999]) is based on problems regarding the CMC in the context of EPR phenomena.

The CMC and its relation to interventionism are not undisputed. ${ }^{54}$ Hausman and Woodward ([1999], [2004a], [2004b]) defend the link between the CMC and manipulation. Cartwright ([2001], [2002]) and Steel ([2006]) offer a critical discussion. It has been also argued that the CMC may fail 
for a number of reasons (see Cartwright [2001], pp. 254-60; Spirtes et al. [2000], pp. 295-7; and Williamson [2005], pp. 52-7). One example is Cartwright's chemical factory ([1999], p. 7). Suppose that a factory $C$ probabilistically produces some chemical $X$, and that when it does produce $X$, it also produces, as a side-effect, a nasty polluant $Y . X$ and $Y$ always occur together (the one is produced if and only if the other is). The factory is a purely probabilistic cause: $X$ (and hence $Y$ ) is produced only $80 \%$ of the time the production process is active. In this set-up, $X$ and $Y$ are not independent conditional on $C .{ }^{55}$ Hence the CMC is violated. Different solutions have been proposed for this problem. For example, Hausman and Woodward ([1999], p. 562) suggest that maybe $C$ has been characterized in insufficient detail. Another possibility is to treat the production of the chemical and its by-product as a single effect, to be modelled by a single variable (cf. Hausman and Woodward [1999], p. 564).

These solutions may be helpful in some cases; yet whether they always work, I do not know. My approach, in any case, would be pragmatic. The CMC should not be considered an a priori principle which is indissolubly tied to the concept of causation. It is a substantive - and hence useful-semantic constraint since it makes causal graphs more than mere dots-and-arrows. But it is also fallible. As a result, when applying causal nets for causal discovery, for causal reasoning, or for representing scientific theories (in a certain context), one always runs the risk that the CMC is violated (in that context). But this is no reason to dispense with the $\mathrm{CMC}$ or with causal nets altogether. Their applicability should be checked on a case-by-case basis. And if it is true that Woodward's interventionism fits many different disciplines, we need not despair. We have good reasons to believe that my causal-structural approach fits many causal scientific 
theories.

\section{Acknowledgements}

I would like to thank Diderik Batens, Gert de Cooman, José Díez, Tjerk Gauderis, Joke Meheus, Sandra Mitchell, Laura Perini, Maarten Van Dyck, Erik Weber, Jon Williamson and two anonymous referees for their helpful comments. Previous versions of this paper were presented at EPSA (Madrid, 2007), the University of Pittsburgh (Center for Philosophy of Science lunchtalk, 2010) and the University of Kent (both at the Centre for Reasoning and the Philosophy Department, 2011). I would like to thank the audiences of these presentations as well.

I am a Postdoctoral Fellow of the Research Foundation - Flanders (FWO - Vlaanderen). Part of the research for this paper was conducted at the Center for Philosophy of Science of the University of Pittsburgh (as a Visiting Fellow) and at the University of Kent (as a Visiting Researcher).

Centre for Logic and Philosophy of Science Department of Philosophy and Moral Sciences

Ghent University

Ghent

Belgium

Bert.Leuridan@Ugent.be

\section{Notes}

${ }^{1}$ Other structuralist accounts of classical genetics can be found in (Balzer and Dawe [1986a], [1986b], [1997]). 
${ }^{2} \mathrm{~A}$ plant is true-breeding for some trait if, when self-fertilized, it only produces offspring with this trait. This definition is not watertight. In a given cross, all the offspring of non-true-breeding plants may, by accident, have the parental phenotype. Yet the larger the set of offspring, the less likely this is.

${ }^{3}$ I will not discuss Woodger's work, since I will not incorporate any of his views below. To some extent, his work also suffers from the common lacuna to be presented in section 4 . Where at first he paid relatively much attention to causation ([1929], passim), he became more skeptical about using causal language in science later on ([1952], p. 194).

${ }^{4}$ The T-theoretical/T-non-theoretical distinction should not be confused with the observable/unobservable distinction. The two distinctions differ both intensionally and extensionally (Díez [2002], p. 15).

${ }^{5}$ Later, Kitcher ([1993], p. 9) rejected thinking of science as a series of theories, defined as sets of statements, while at the same time sticking to the view that explanatory schemata or patterns are implicit in scientific practice ([1993], pp. 82-4).

${ }^{6}$ See also (Balzer and Dawe [1986a], pp. 58 and 62) and (Balzer and Dawe [1986b], p. 179) for a similar reluctance to use causal language.

${ }^{7}$ Woodward ([2003], pp. 360-2) criticizes Kitcher's theory as a theory of causation and explanation. For criticisms of Kitcher's theory as a theory of unification and for my solution to the problems raised, see section 18 .

${ }^{8}$ I would like to thank an anonymous referee for pressing me on this issue.

${ }^{9}$ Causation is also deemed by many to play an important role in policy, manipulation or intervention. The consensus view in philosophy of science now is that causal relations are potentially exploitable for manipulation, 
policy and control, whereas spurious correlations are not; see for example (Cartwright [1983]; Spirtes et al. [2000]; Pearl [2000]; Woodward [2003]; and Williamson [2005]). However, see (Leuridan et al. [2008]) for a specific type of policy - selective policy - that is based on spurious correlations such as the phenotypic distributions studied in classical genetics.

${ }^{10}$ For reasons of space, I will not systematically discuss the other accounts of causation that are available on the philosophical market. The two reasons to be discussed should suffice to show at least that Woodward's theory is $a$ well-suited candidate for my purposes.

${ }^{11}$ In this definition, 'independent' means 'probabilistically independent according to $P^{\prime}$.

${ }^{12}$ The Causal Markov Condition does not rule out that $P$ satisfies some extra conditional or unconditional independence relations as well. To simplify automated causal discovery, many search algorithms invoke another, complementary assumption (Faithfulness) which rules out such extra independence relations, see (Spirtes et al. [2000], p. 31). As I will not touch upon the problem of causal discovery, I will not require that $P$ and $G$ are faithful.

${ }^{13}$ When I will apply the definitions developed in this section and the next one to the theory of classical genetics, I will not attach significance to the order of the values in the elements of $[U]$ and hence will use a relaxed notation.

${ }^{14} \mathrm{~A}$ bijection or bijective function $f$ is a function which is both injective (if $f(x)=f(y)$, then it must be the case that $x=y$ ) and surjective (for every element $w$ in its range, there is an element $v$ in its domain such that $w=f(v))$. Source: http://mathworld.wolfram.com.

${ }^{15}$ Originally, credal nets were used to facilitate reasoning with imprecise 
probabilities (Cozman [2000]). This is not my aim in this paper.

${ }^{16}$ In the case of self-fertilization, the paternal and the maternal group are the same. Still, it is convenient to represent such crosses by means of the causal graph in figure 1.

${ }^{17}$ Morgan does not use 'tall-producing allele' or 'short-producing allele'. But his presentation of inheritance of flower-colour in four-o'clocks is phrased in terms of 'white-producing gene' and 'red-producing gene' (Morgan [1928], p. 5-7).

${ }^{18}$ The combination principle should not be confused with the COMBINATOR function in (Balzer and Lorenzano [2000]). In their paper, the role of the gametes is not made explicit.

${ }^{19}$ Chance fertilization comes down to $G C_{1}$ and $G C_{2}$ being probabilistically independent, which is true for any probability distribution that satisfies the Causal Markov Condition relative to figure 1 (or to any isomorphic graph), as can be seen by means of the $d$-separation criterion, see (Pearl [2000], p. 16; Spirtes et al. [2000], p. 44).

${ }^{20}$ Strictly speaking, what is explained in classical genetics is not the data, but what Bogen and Woodward ([1988], pp. 305-6) call 'phenomena'. Phenomena are relatively stable and repeatable and can hence be predicted and explained by theories. Data are idiosyncratic to a particular experimental setting and hence not predictable or systematically explainable. For example, the phenotypic 3:1-ratio of tall versus short in the $F_{2}$ generation of cross 2 (section 8.1) is a phenomenon, an idealized statistical generalization. Mendel's data for that cross were $\frac{787}{1064}$ versus $\frac{277}{1064}$, which is close but not identical to $3: 1$ ([1965], p. 13). From here onwards, by 'data' I will mean 'phenomena' in the above sense.

${ }^{21}$ Within the structuralist literature, there is some discussion as to 
whether all explanation has to be 'ampliative', i.e. whether it always requires the addition of $\mathbf{T}$-theoretical concepts. I would like to thank José Díez for pointing this out.

${ }^{22} \mathrm{Abduction}$ is a type of inference which is to be distinguished from 'deduction' and 'induction' (Douven [2011], section 1.1). Peirce ([CP], 5.189) defines 'abduction' as follows: 'The surprising fact, C, is observed; But if $\mathrm{A}$ were true, $\mathrm{C}$ would be a matter of course, Hence, there is reason to suspect that A is true.' In classical genetics, the inference from phenotypes to genotypes was explicitly abductive and, given the CG-theoretical nature of the $G T_{i}$ and $G C_{i}$ variables, based on the explanatory principles of genetics itself. This does not mean, however, that the explanation being given is ad hoc. The explanatory principles were expected to hold in a wider range of related cases (see section 18 for an explication of this 'wider range of related cases') and they were explicitly tested by means of test crosses (see cross 3 below). In Waters' words: they were 'local inferences to the best explanation' ([2004], p. 802). (Inference to the best explanation is a close nephew of abduction. A hypothesis $A$ is the best explanation for $C$ if it outdoes all other explanations for $C$.)

${ }^{23} \mathrm{~A}$ reciprocal variant of cross 1 , in which the pollen plants are short and the egg plants are pure tall, gives the same results, as Mendel ([1865], p. 9) discovered. These results can be explained by means of the very same set of principles: there is a $P \in \mathbb{P}^{1}$ such that $P\left(P T_{1}=\right.$ short $)=\left(P T_{2}=\right.$ tall $)=1.00$, and $P\left(G T_{1}=s s\right)=P\left(G T_{2}=t t\right)=1.00$. Given that $P \in \mathbb{P}^{1}$, $P\left(P T_{3}=\right.$ tall $)=1.00$. This also corresponds to the data. In cases of sex-linked inheritance, however, reciprocal crosses do not result in identical filial phenotypic distributions. Such cases can also be modelled in my framework, but I will not do so here. 
${ }^{24}$ Where $U=\left\{A_{1}, \ldots, A_{n}\right\} \subseteq V$ and $b: V \rightarrow V^{\prime}$, let $b(U)=\{b(A) \mid A \in U\}$ and $b^{\prime}([U])=\left[b\left(A_{1}\right)\right] \times \ldots \times\left[b\left(A_{n}\right)\right]$.

${ }^{25}$ Generic credal nets not only resemble Darden's abstract explanatory pattern. They also resemble the structuralist notion of 'theory-element'. Moreover, they also resemble van Fraassen's 'model types' (see van Fraassen [1980], p. 44 and especially Lloyd [1983], pp. 118-21). However, my account of explanation is more substantive (or less skeptical) than van Fraassen's ([1980], chapter 5) and Lloyd's ([1983], pp. 116-7), without being a truly general account of explanation (see sections 9 and 17; for a defense of truly general accounts of explanation, see Nickel [2010]; for a critique of his arguments, see Díez et al. [forthcoming]).

${ }^{26} \mathrm{My}$ framework cannot account for every single detail of Darden's analysis. For example, Darden ([1991], pp. 195-9) strongly clings to the role of diagrammatic representations (e.g. pedigree diagrams) in the history of classical genetics. Given that diagrams, and more broadly visual representations, may play an important role in functional explanation (Perini [2005a]), in scientific arguments (Perini [2005b]), and in confirmation (Perini [2005c]), all of which are highly important in science, the causal-structural account should be viewed as complementary to (rather than a strict alternative for) Darden's analysis.

${ }^{27}$ Darden's ([2005]) main interest is in the relation between classical genetics and molecular biology. She argues that these fields investigated different, serially integrated, hereditary mechanisms and that molecular biology offered a kind of explanatory extension of the field of classical genetics, albeit via mechanism schemas instead of Kitcherian argument patterns (Darden [2005], p. 350). For an interesting critique of the explanatory extension view, based on the existence of 'explanatory 
interference' between the two fields, see (Baetu [2011]).

${ }^{28} \mathrm{I}$ would like to thank both anonymous referees for pressing me on this issue.

${ }^{29}$ Morgan does not use $r$ and $w$ (but black and white circles) for the redand white-producing alleles in his diagrammatic representation of these crosses (Morgan [1928], p. 7).

${ }^{30}$ The reverse assumption would result in a different $\mathcal{B} \in \mathfrak{B}^{3}$.

${ }^{31}$ Note that $P^{1}\left(V^{1}\right)$ and $P^{1}\left(V^{3}\right)$ are different distributions, given that $V^{1}$ and $V^{3}$ are different sets of variables (they are not even value-isomorphic). Likewise, $\mathcal{B}^{1}=\left\langle G^{3}, P^{1}\right\rangle$ with $P^{1} \in \mathbb{P}^{3}$ should not be confused with $\mathcal{B}^{1}=\left\langle G^{1}, P^{1}\right\rangle$ with $P^{1} \in \mathbb{P}^{1}$.

${ }^{32}$ Monohybrid crosses with codominance, instead of incomplete dominance, can also be represented by causal nets that are distribution-identical to some member of $\mathfrak{B}^{\beta}$. This is in line with the fact that to distinguish between codominance and incomplete dominance, one has to invoke a theory (at the molecular level) other than classical genetics. In (Morgan [1928]), no exemplar of codominance is mentioned.

${ }^{33}$ See also (Kitcher [1993], pp. 256-63) for a more elaborate account of anomaly-resolution in terms of the deletion/alteration of patterns (or 'constraints') in an escape tree. Darden ([1991]) does not discuss the status of incomplete dominance as a model anomaly.

${ }^{34}$ The status of the principle of complete dominance was hotly debated in the early days of classical genetics. The question was whether it is a universal law. Mendel ([1865]) only discussed crosses with complete dominance but there is no clear indication that he deemed complete dominance a universal phenomenon - quite to the contrary. Nevertheless De Vries ([1900], p. 110) took it to be a (nearly) universal phenomenon. 
Correns ([1900], pp. 122 and 132), by contrast, mentioned several failures of complete dominance. Weldon ([1902], pp. 229, 236-52) attributed the 'Law of Dominance' a central place in Mendel's theory and showed that it was plagued by exceptions. Bateson ([1902], pp. 117-8) replied to Weldon's arguments by denying the principle of complete dominance this central place. Hence exceptions to it should not count heavily against the theory of Mendelian genetics.

${ }^{35}$ Mendel ([1865], p. 17-23) also performed multihybrid crosses with three or more characters and independent assortment. Morgan ([1928], p. 10) mentions these only in passing (but see Morgan [1919], pp. 71-2 for an example). Crosses with three or more independent pairs of traits can be easily incorporated in my framework, but I will not do it here.

${ }^{36}$ For the extensions of $\left[G T_{i}\right]$ and $\left[G C_{i}\right]$, see (Morgan [1928], p. 9, figure 8).

${ }^{37}$ The text in (Morgan [1928], p. 11, figure 9) gives the impression that the cross concerned purple and white flowers, instead of purple and red ones. This conflicts with Morgan's main text and with (Darden [1991], p. 122).

${ }^{38}$ Morgan ([1928], p. 11) uses pictorial elements instead of letters to denote these alleles.

${ }^{39}$ The calculations for the other phenotypes are somewhat more elaborate, given the multiple realizability of dominant phenotypic traits.

${ }^{40}$ Morgan does not discuss this particular cross, but an analogous way of reasoning can be found in (Morgan [1928], pp. 16-7).

${ }^{41}$ The if-direction has been illustrated by means of the data from Bateson and Punnett. For the only if-direction, suppose first that $G T_{i}=G G W W$ or any other doubly homozygous value. Then (absent 
mutation) only one kind of gametes can be produced, in casu $G C_{i}=G W$. Second, suppose that $G T_{i}=G G W w$ or any other singly homozygous value. Then one chromosome will carry $G$ and $W$. The other chromosome will carry $G$ and $w$. Part of the gametes (say, $x \%$ ) will not be the result of crossing-over. Half of them $\left(\frac{x}{2} \%\right)$ will be $G W$, the other half will be $G w$. The rest of the gametes, $(100-x) \%$, will be the result of crossing-over. Half of these, $\frac{100-x}{2} \%$, will be $G w$; the other half will be $G W$. Consequently, $\frac{x}{2}+\frac{100-x}{2}=50 \%$ of the gametes will be $G W$, the other half will be $G w$. Hence, the gametes of both doubly homozygous plants and singly heterozygous plants are as in the case of independent assortment (even though they result from strongly different underlying mechanisms).

${ }^{42}$ Sturtevant equated one map unit $(\mathrm{mu})$ with 1 percent recombination. In honour of Morgan's work, map units are often referred to as centimorgans (cM). (Klug et al. [2006], p. 105) Map distances are not absolute distances, since not all parts of the chromosome are evenly prone to interchange. Moreover, a crossover event in one region of the chromosome may inhibit a second event in nearby regions (positive interference). Positive interference increases as the genes in question are closer. This may be explained by physical constraints preventing the formation of closely aligned chiasmata. (Klug et al. [2006], p. 114)

${ }^{43}$ Obviously, cases of incomplete dominance, codominance, etc. also conform to Morgan's observation, but the corresponding dihybrid crosses are represented by generic credal nets other than $\mathfrak{B}^{\delta}$.

${ }^{44}$ Stability is a gradual notion. It pertains to the conditions upon which a regularity is contingent. Strength also is a gradual notion. It can be thought of in terms of covariance or correlation, with deterministic regularities being a limit case. (Leuridan [2010], pp. 324-5; Mitchell [1997], 
pp. S477-8, [2000], pp. 259-63)

${ }^{45}$ Although classical genetics' causal nets are possible realizations in which all of the theory's valid sentences are satisfied (cf. Suppes [1969], p. 24), and hence 'things depicted by classical genetics', cf. (Balzer et al. [1987], p. 2), they are themselves abstract representations of the real-world states of affairs.

${ }^{46}$ One pattern is more stringent than another one if the conditions it sets on instantiations are more difficult to satisfy (Kitcher [1989], p. 433).

${ }^{47}$ See also (Mäki [1990]; Marchionni [2005]). Woodward ([2003], p. 362) calls this kind of unification physical unification, but he and Mäki do not refer to each other's writings.

${ }^{48}$ The notion of ontological unification is not wedded to an ontic account of explanation, as is evident from Mäki's use of 'the referential and representational capabilities of theories'. Ontological unification and ontic explanation should not be conflated.

${ }^{49}$ Alleles and genotypes may be considered properties (of organisms) instead of objects. Then in a sense Kitcher's schematic sentence 'There are two alleles $A, a$. $A$ is dominant, $a$ is recessive.' is a second-order sentence. I write 'in a sense', since the quantifier indirectly refers to filling instructions ( $A$ and $a$ are dummy letters, not common second order variables).

${ }^{50}$ Credal nets here are defined in terms of Woodwardian causal relations (section 6) and laws or explanatory principles that can be conceived of in the sense of Mitchell's pragmatic laws (section 17), not in terms of mechanisms. I have argued elsewhere, however, that there need not be any tension between the former and the latter (Leuridan [2010], section 7).

${ }^{51}$ This claim should be nuanced. (I would like to thank an anonymous referee for pressing me on this issue.) Figure 1 represents only certain 
aspects of this common causal mechanism, to wit the qualitative relations between those properties of the entities, processes, and powers that can be represented by the variables $G T_{1}, \ldots, P T_{3}$. That is not surprising: all representations are abstractions that leave out part of their subject matter.

${ }^{52}$ For a broader conception of the domain of classical genetics, see (Waters [2004]).

${ }^{53}$ This same intuition can be found in the works of Bartelborth ([1996], [2002]), Sintonen ([1989]) and Lloyd ([1983]).

${ }^{54}$ I will not discuss the tenability of the Faithfulness Condition, as I did not assume it.

${ }^{55}$ If $X$ and $Y$ would be independent conditional on $C$, then $P(x y \mid c)=P(x \mid c) \times P(y \mid c)$ for all $x \in[X], y \in[Y]$ and for all $c \in[C]$ (provided $P(c) \neq 0)$. In this case, however, $P(X=1, Y=1 \mid C=1)=$ $0.8 \neq P(X=1 \mid C=1) \times P(Y=1 \mid C=1)=0.64$ (where ' $X=1$ ' means that $X$ is present, ' $Y=1$ ' means that $Y$ is present, and ' $C=1$ ' means that the production process is active).

\section{References}

Baetu, T. M. [2011]: 'Mechanism schemas and the relationship between biological theories', in P. Illari, F. Russo and J. Williamson (eds), 2011, Causation in the Sciences, Oxford: Oxford University Press, pp. 407-24.

Balzer, W. and Dawe, C. [1986a]: 'Structure and comparison of genetic theories I', The British Journal for the Philosophy of Science, 37(1), pp. $55-69$.

Balzer, W. and Dawe, C. [1986b]: 'Structure and comparison of genetic 
theories II', The British Journal for the Philosophy of Science, 37(2), pp. 177-91.

Balzer, W. and Dawe, C. [1997]: Models for Genetics, Frankfurt: Peter Lang.

Balzer, W. and Lorenzano, P. [2000]: 'The logical structure of classical genetics', Journal for General Philosophy of Science, 31(2), pp. 243-66.

Balzer, W., Moulines, C. U., and Sneed, J. D. [1987]: An Architectonic for Science. The Structuralist Program, Dordrecht: Reidel.

Balzer, W., Sneed, J. D., and Moulines, C. U. (eds) [2000]: Structuralist Knowledge Representation: Paradigmatic Examples, Amsterdam: Rodopi.

Bartelborth, T. [1996]: 'Scientific explanation', in W. Balzer and C. Moulines (eds), 1996, Structuralist Theory of Science, Berlin: de Gruyter, pp. 23-43.

Bartelborth, T. [2002]: 'Explanatory unification', Synthese, 130, pp. 91-107.

Bateson, W. [1900]: 'Problems of heredity as a subject for horticultural investigation', Journal for the Royal Horticultural Society, 25, pp. 54-61, electronically reprinted by the Electronic Scolarly Publishing Project (<www.esp.org $>$; page numbers ranging 1-10).

Bateson, W. [1902]: Mendel's Principles of Heredity. A Defence, London: Cambridge University Press.

Bechtel, W. [2011]: 'Mechanism and biological explanation', Philosophy of Science, 78(4), pp. 533-57. 
Bechtel, W. and Abrahamsen, A. [2005]: 'Explanation: a mechanist alternative', Studies in History and Philosophy of Biological and Biomedical Sciences, 36, pp. 421-41.

Bogen, J. and Woodward, J. [1988]: 'Saving the phenomena', The Philosophical Review, XCVII(3), pp. 303-52.

Bryant, W. [2010]: General Equilibrium. Theory and Evidence, Singapore: World Scientific Publishing.

Cartwright, N. [1983]: How the Laws of Physics Lie, Oxford: Clarendon Press.

Cartwright, N. [1999]: 'Causal diversity and the Markov condition', Synthese, 121, pp. 3-27.

Cartwright, N. [2001]: 'What is wrong with Bayes nets?', The Monist, 84(2), pp. 242-64.

Cartwright, N. [2002]: 'Against modularity, the causal Markov condition, and any link between the two: Comments on Hausman and Woodward', The British Journal for the Philosophy of Science, 53, pp. 411-53.

Casini, L., McKay Illari, P., Russo, F., and Williamson, J. [2011]: 'Models for prediction, explanation and control: Recursive Bayesian Networks', Theoria. An International Journal for Theory, History and Foundations of Science, 70, pp. 5-33.

Clarke, B., Leuridan, B., and Williamson, J. [unpublished]: 'Modelling mechanisms with causal cycles', Unpublished manuscript.

Correns, C. [1900]: 'G. Mendel's Laws concerning the behavior of progeny of varietal hybrids', in C. Stern and E. R. Sherwood (eds), 1966, The 
Origin of Genetics: A Mendel Source Book, San Francisco: Freeman, pp. 119-32.

Cozman, F. G. [2000]: 'Credal networks', Artificial Intelligence, 120, pp. 199-233.

Cozman, F. G. [2005]: 'Graphical models for imprecise probabilities', International Journal of Approximate Reasoning, 39, pp. 167-84.

Craver, C. F. [2007]: Explaining the Brain: Mechanisms and the Mosaic Unity of Neuroscience, Oxford: Clarendon Press.

Darden, L. [1991]: Theory Change in Science: Strategies from Mendelian Genetics, Oxford: Oxford University Press.

Darden, L. [2002]: 'Strategies for discovering mechanisms: Schema instantiation, modular subassembly, forward/backward chaining', Philosophy of Science, 69, pp. S354-65.

Darden, L. [2005]: 'Relations among fields: Mendelian, cytological and molecular mechanisms', Studies in History and Philosophy of Biological and Biomedical Sciences, 36, pp. 349-71.

Darden, L. and Rada, R. [1988]: 'Hypothesis formation using part-whole interrelations', in D. Helman (ed.), 1988, Analogical Reasoning, Dordrecht: Reidel, pp. 341-75.

de Vries, H. [1900]: 'The law of segregation of hybrids. [Das Spaltungsgesetz der Bastarde (Preliminary communication)]', in C. Stern and E. R. Sherwood (eds), 1966, The Origin of Genetics: A Mendel Source Book, San Francisco: Freeman, pp. 107-17. 
Díez, J. A. [2002]: 'A program for the individuation of scientific concepts', Synthese, 120, pp. 13-48.

Díez, J. A., Khalifa, K., and Leuridan, B. [forthcoming]: 'General theories of explanation: buyer beware', Synthese, forthcoming.

Douven, I. [2011]: 'Abduction', in E. N. Zalta (ed.), The Stanford Encyclopedia of Philosophy, <plato.stanford.edu >.

Dowe, P. [2000]: Physical Causation, Cambridge: Cambridge University Press.

Earman, J., Glymour, C., and Mitchell, S. [2002]: 'Ceteris paribus lost', Erkenntnis, 57(3), pp. 281-301.

Forge, J. [2002]: 'Reflections on structuralism and scientific explanation', Synthese, 130, pp. 109-21.

Gijsbers, V. [2007]: 'Why unification is neither necessary nor sufficient for explanation', Philosophy of Science, 74(4), pp. 481-500.

Glennan, S. S. [1996]: 'Mechanisms and the nature of causation', Erkenntnis, 44, pp. 49-71.

Glennan, S. S. [2002]: 'Rethinking mechanistic explanation', Philosophy of Science, 69(3), pp. S342-53.

Hamminga, B. and Balzer, W. [2000]: 'The basic structure of neo-classical general equilibrium theory', in W. Balzer, J. D. Sneed and C. U. Moulines (eds), 2000, Structuralist Knowledge Representation: Paradigmatic Examples, Amsterdam: Rodopi, pp. 149-70.

Hausman, D. M. [1998]: Causal Asymmetries, Cambridge: Cambridge University Press. 
Hausman, D. M. and Woodward, J. [1999]: 'Independence, invariance and the causal Markov condition', The British Journal for the Philosophy of Science, 50, pp. 521-83.

Hausman, D. M. and Woodward, J. [2004a]: 'Manipulation and the causal Markov condition', Philosophy of Science, 71, pp. 846-56..

Hausman, D. M. and Woodward, J. [2004b]: 'Modularity and the causal Markov condition: a restatement', The British Journal for the Philosophy of Science, 55, pp. 147-61.

Hempel, C. G. [1965]: Aspects of Scientific Explanation and Other Essays in the Philosophy of Science, New York: The Free Press.

Kitcher, P. [1981]: 'Explanatory unification', Philosophy of Science, 48(4), pp. 507-31.

Kitcher, P. [1989]: 'Explanatory unification and the causal structure of the world', in P. Kitcher and W. C. Salmon (eds), 1989, Scientific Explanation., volume XIII of Minnesota Studies in the Philosophy of Science., Minneapolis: University of Minnesota Press, pp. 410-505.

Kitcher, P. [1993]: The Advacement of Science. Science without Legend, Objectivity without Illusions, New York: Oxford University Press.

Kitcher, P. and Salmon, W. C. (eds) [1989]: Scientific Explanation., volume XIII of Minnesota Studies in the Philosophy of Science. Minneapolis: University of Minnesota Press.

Klug, W., Cummings, M., and Spencer, C. [2006]: Concepts of Genetics, New Jersey: Pearson Prentice-Hall, 8th edition. 
Kuhn, T. S. [1962 (1996)]: The Structure of Scientific Revolutions, Chicago: University of Chicago Press, 3rd edition.

Lazebnik, Y. [2002]: 'Can a biologist fix a radio? - or, what I learned while studying apoptosis', Cancer Cell, 2, pp. 179-82.

Leuridan, B. [2007]: 'Galton's blinding glasses. Modern statistics hiding causal structure in early theories of inheritance', in F. Russo and J. Williamson (eds), 2007, Causality and Probability in the Sciences, Texts In Philosophy series, London: College Publications, pp. 243-62.

Leuridan, B. [2010]: 'Can mechanisms really replace laws of nature?', Philosophy of Science, 77, pp. 317-40.

Leuridan, B., Weber, E., and Van Dyck, M. [2008]: 'The practical value of spurious correlations: Selective versus manipulative policy', Analysis, 68, pp. 298-303.

Lloyd, E. A. [1983]: 'The nature of Darwin's support for the theory of natural selection', Philosophy of Science, 50(1), pp. 112-29.

Machamer, P., Darden, L., and Craver, C. F. [2000]: 'Thinking about mechanisms', Philosophy of Science, 67(1), pp. 1-25.

Mäki, U. [1990]: 'Scientific realism and austrian explanation', Review of Political Economy, 2(3), pp. 310-44.

Mäki, U. [2001]: 'Explanatory unification : double and doubtful', Philosophy of the Social Sciences, 31(4), pp. 488-506.

Mancosu, P. [2011]: 'Explanation in mathematics', in E. N. Zalta (ed.), The Stanford Encyclopedia of Philosophy, < plato.stanford.edu>. 
Marchionni, C. [2005]: 'Unity, plurality and explanation. the case of geographical economics and its neighbours', Unpublished PhD thesis.

Mendel, G. [1865]: 'Experiments on plant hybrids', in C. Stern and E. R. Sherwood (eds), 1966, The Origin of Genetics: A Mendel Source Book, San Francisco: Freeman, pp. 1-48.

Mendel, G. [1866 (1933)]: Versuche über Pflanzenhybriden, Ostwald's Klassiker der Exakten Wissenschaften, Leipzig: Akademische Verlagsgesellschaft.

Mitchell, S. D. [1997]: 'Pragmatic laws', Philosophy of Science, 64(4, supplement), pp. S468-79.

Mitchell, S. D. [2000]: 'Dimensions of scientific law', Philosophy of Science, 67(4), pp. 242-65.

Mitchell, S. D. [2002]: 'Ceteris paribus - an inadequate representation for biological contingency', Erkenntnis, 57(3), pp. 329-50.

Morgan, T. H. [1919]: The Physical Basis of Heredity, Philadelphia: Lippincott.

Morgan, T. H. [1926 (1928)]: The Theory of the Gene, New Haven: Yale University Press, revised and enlarged edition.

Nickel, B. [2010]: 'How general do theories of explanation need to be?', Nô̂s, 44(2), pp. 305-28.

Pearl, J. [2000]: Causality. Models, Reasoning, and Inference, Cambridge: Cambridge University Press.

Peirce, C. S. [1931-1958]: Collected Papers of Charles Sanders Peirce, Cambridge, MA: Harvard University Press. 
Perini, L. [2005a]: 'Explanation in two dimensions: Diagrams and biological explanation', Biology and Philosophy, 20, pp. 257-69.

Perini, L. [2005b]: 'The truth in pictures', Philosophy of Science, 72(1), pp. $262-85$.

Perini, L. [2005c]: 'Visual representations and confirmation', Philosophy of Science, 72(5), pp. 913-26.

Pietroski, P. and Rey, G. [1995]: 'When other things aren't equal: Saving ceteris paribus laws from vacuity', The British Journal for the Philosophy of Science, 46, pp. 81-110.

Salmon, W. C. [1984]: Scientific Explanation and the Causal Structure of the World, Princeton, NJ: Princeton University Press.

Sintonen, M. [1989]: 'Explanation: In search of the rationale', in P. Kitcher and W. C. Salmon (eds), 1989, Scientific Explanation., volume XIII of Minnesota Studies in the Philosophy of Science., Minneapolis: University of Minnesota Press, pp. 253-82.

Spirtes, P., Glymour, C., and Scheines, R. [2000]: Causation, Prediction, and Search, Cambridge, MA: MIT Press.

Steel, D. [2006]: 'Comment on Hausman \& Woodward on the causal Markov condition', British Journal for the Philosophy of Science, 57(1), pp. 219-31.

Stern, C. and Sherwood, E. R. (eds) [1966]: The Origin of Genetics: A Mendel Source Book, San Francisco: Freeman.

Strevens, M. [2004]: 'The causal and unification approaches to explanation unified - causally', Nô̂s, 38(1), pp. 154-76. 
Strevens, M. [2008]: Depth, Cambridge, MA: Harvard University Press.

Suárez, M. and San Pedro, I. [2011]: 'Causal Markov, robustness and the quantum correlations', in M. Suárez (ed.), 2011, Probabilities, Causes and Propensities in Physics, volume 347 of Synthese Library, Dordrecht: Springer, pp. 173-93.

Suppes, P. (ed.) [1969]: Studies in the Methodology and Foundations of Science. Selected Papers from 1951 to 1969, Dordrecht - Holland: D. Reidel Publishing Company.

van Fraassen, B. [1980]: The Scientific Image, Oxford: Clarendon Press.

Waters, C. K. [1994]: 'Genes made molecular', Philosophy of Science, 61(2), pp. 163-85.

Waters, C. K. [2004]: 'What was classical genetics?', Studies in History and Philosophy of Science, 35, pp. 783-809.

Waters, C. K. [2007]: 'Causes that make a difference', The Journal of Philosophy, CIV(11), pp. 551-79.

Weldon, W. [1902]: 'Mendel's laws of alternative inheritance in peas', Biometrika, 1, pp. 228-54.

Williamson, J. [2005]: Bayesian Nets and Causality. Philosophical and Computational Foundations, New York: Oxford University Press.

Woodger, J. H. [1929]: Biological Principles. A Critical Study. London: Routledge \& Kegan Paul Ltd.

Woodger, J. H. [1952]: Biology and Language. An Introduction to the Methodology of the Biological Sciences including Medicine, Cambridge: Cambridge University Press. 
Woodger, J. H. [1959]: 'Studies in the foundations of genetics', in

L. Henkin, P. Suppes and A. Tarski, (eds), 1959, The Axiomatic Method, Amsterdam: North-Holland.

Woodward, J. [2002]: 'There is no such thing as a ceteris paribus law', Erkenntnis, 57(3), pp. 302-28.

Woodward, J. [2003]: Making Things Happen. A Theory of Causal Explanation, New York: Oxford University Press.

Woodward, J. [2009]: 'Scientific explanation', in E. N. Zalta (ed.), The Stanford Encyclopedia of Philosophy, < plato.stanford.edu >. 